\title{
Design of Reduced-Order Multiple Observers for Uncertain Systems with Unknown Inputs
}

\author{
Mihai Lungu (i) \\ Faculty of Electrical Engineering, University of Craiova, 107 Decebal Blvd., Craiova, Romania \\ Correspondence should be addressed to Mihai Lungu; lma1312@yahoo.com
}

Received 28 October 2017; Accepted 12 March 2018; Published 14 May 2018

Academic Editor: Arturo Buscarino

Copyright (C) 2018 Mihai Lungu. This is an open access article distributed under the Creative Commons Attribution License, which permits unrestricted use, distribution, and reproduction in any medium, provided the original work is properly cited.

\begin{abstract}
The paper presents the design of a new reduced-order multiple observer for the estimation of the state associated with TakagiSugeno systems with unknown inputs, this being only the second reduced-order multiple observer ever designed. The design of reduced-order multiple observers which can achieve the finite-time state reconstruction for nonlinear systems described by multiple models is a niche area problem; the author of this paper continuing his work started with the introduction of the reducedorder multiple observer concept. The new multiple observer is a combination of a typical reduced-order observer for linear-time invariant multivariable systems and a full-order multiple observer for Takagi-Sugeno systems. The sufficient stability conditions of the observer are derived via the Lyapunov theory and its robustness is improved by means of a novel and efficient method which cancels the negative effect of the uncertainties appearing in the system. To validate the suggested design algorithm, the steps of the design procedure have been summarized and software implemented for the concrete case of a light aircraft lateral-directional motion.
\end{abstract}

\section{Introduction}

In many real-world applications, there are difficulties in obtaining the measurement of the state variables describing the functioning of a system; sometimes, this is even impossible because of the physical constraints and/or economical restrictions; the usage of observers instead of sensors is a solution largely adopted in order to avoid these problems. The state reconstruction is also affected by some unmeasurable or inaccessible inputs (denoted as unknown inputs), which can be at the origin of biased estimations if these unknown inputs are not correctly taken into consideration [1-3]. The estimation of the states and unknown inputs (noises, measurement uncertainties, faults of sensors or actuators, etc.) for a physical system is needed in order to conceive a control strategy able to minimize the negative effects of the disturbances $[4,5]$. There are differences between the observers designed for linear or nonlinear systems, the design process being more difficult in the second case; to overcome this drawback, viable solutions can be the model order reduction $[6,7]$ or the usage of the linearization method to obtain a linear system, because this technique (detailed in [8]) allows the transformation of any nonlinear system into the so-called multiple model-sum of linear models, each of them characterizing the system in a specific operating regime. The principle of the multiple model approach is based on the reduction of the system's complexity by the decomposition of its operating space in a finite number of operating zones [9]; one obtains local models which are linear, affine, and time invariant systems due to the presence of some linearization constants. The relative contribution of each submodel is quantified by a weighting function. Initially, the multiple observers have been used for failure detection $[10,11]$ and switching control [12], where the multiple observers are designed for candidate plant models. In the research area of multiple models' estimation, the aim of the paper [13] was the state estimation for perturbed output nonlinear systems; a Lyapunov based sliding mode multiple observer is presented, the proof of the observer stability being ensured by a suitable choice of estimation gains-solutions of linear matrix inequalities (LMIs). In [14], the authors focus on the state estimation of a nonlinear system described by a Takagi-Sugeno (T-S) multiple model having unknown inputs and outputs; the proposed approach consists of a mathematical transformation which enables 
the "conversion" of the unknown outputs into "pseudo" unknown inputs-eliminated through the designed multiple observer. In [15], a joint state and parameter estimation observer, proposed for T-S systems whose matrices depend on unknown parameters, is applied to a system model for a heat-exchanger-zones combination and the implementation challenges; the unknown parameters are rewritten using the sector nonlinearity approach through weighting functions and sector extreme values; also, an $L_{2}$ formulation is used to handle the situation arising out of the difference between the actual and estimated weighting functions. Almost all papers dealing with the design of multiple observers assume that the weighting functions depend on measurable variables, few works being devoted to the case of nonmeasurable decision variables [3]; the paper [16] proposes a method which reduces the conservatism of the precedent works by reducing the number of linear matrix inequalities to be solved and relaxing the conditions for this method to be applied. Another multiple observer has been designed in [8]; it uses the interpolation principle for local observers and the calculation of the multiple observer gains is used to calculate the gains of the local observers. The output feedback controller's design for Takagi-Sugeno fuzzy system with timevarying state-delay has been investigated in [17]. Also, a great amount of research works has been carried out on application of T-S fuzzy systems for networked nonlinear systems and fuzzy stochastic systems [18].

In all works from literature dealing with the design of multiple observers for the estimation of states and unknown inputs, the designed observers are full-order multiple observers (FOMOs); until now, there is only one paper in the literature (paper [19]) dealing with the design of a reduced-order multiple observer (ROMO) for Takagi-Sugeno systems with unknown inputs; the observer belongs to the author of this paper who introduced for the first time the concept of reduced-order multiple observer, the design of such observers being, therefore, a niche research theme. The main advantage of the reduced-order multiple observers is related to the decrease of the system sensors' number: some of the state variables are measured, while the others are estimated [19]; moreover, all the other advantages of general reduced-order observers can be considered advantages of ROMOs with respect to FOMOs. The main drawbacks of the ROMO from [19] are (1) the large number of existence conditions; (2) the usage of pole placement method because it can lead to the undesired phenomena called the eigenvalues' sensitivity; (3) lack of solutions for robustness' improvement in the case of uncertainties in the system; (4) high convergence time for the estimation error. The ROMO designed in this paper has smaller number of existence conditions, does not use the pole placement technique, and includes an innovative algorithm to increase its robustness with respect to uncertainties; moreover, it will be shown that the problem of ROMO's design for T-S unknown input systems can be reduced to a standard one (the unknown inputs do not interfere in the equations of the observer).

The observer designed in this paper (the second ROMO ever obtained) is a mixture of the approaches presented in $[4,20,21]$, improved by a rigorous method to increase its robustness. In [20], the necessary and sufficient conditions are presented for the existence and the design of an unknown input functional observer for linear-time invariant (LTI) multivariable systems, subjected to unknown inputs, the existence of the observer being verified by computing a nullspace of a known matrix and testing some matrices' rank conditions. In [21], a full-order observer without unknown inputs reconstruction is suggested in order to achieve the finite-time reconstruction of the state vector for a class of unknown inputs LTI systems, the observer design approach being based on an innovative method to obtain a fullorder observer with no existence conditions. The paper [9] presents the design methodology of a FOMO associated with Takagi-Sugeno descriptor systems, a method to improve the observer robustness being also provided. In the present paper, the approaches from these three papers are partially used and additional and innovative new elements within the design approach are brought: the new form of the reducedorder observer, a new method for solving matrix equation systems with more unknowns than equations, an innovative method to improve the observer's robustness with respect to uncertainties, and a "while" loop based method to increase the new ROMO's generality and applicability, as well as to decrease its existence conditions' number.

The paper is organized as follows: the structure of a general multiple model is presented in Section 2; the design methodology of the new reduced-order multiple observer is provided in Section 3; in the next section, the new ROMO's robustness is improved by means of robustness terms introduced in the dynamics of the observer; a summarization of all the design approach steps is achieved in the fifth section, while in Section 6 the new observer is validated by means of numerical simulations for the concrete case of a light aircraft lateral-directional motion during landing; a brief comparison between the only two existing ROMOs (the one designed in this paper and the one from [19]) as well as between the new ROMO and other FOMOs from literature is also achieved. Finally, some conclusions are shared in Section 7.

\section{Structure of the Takagi-Sugeno Multiple Model}

Depending on the connections existing between its local models, two types of multiple models can be distinguished: (1) multiple models having homogeneous submodels (the local models have the same state) and (2) multiple models having heterogeneous submodels (the local models have not the same state); the first variant is more commonly used, being introduced by Takagi and Sugeno in the fuzzy modeling [22] and by Johansen and Foss in the modeling using the concept of multiple model [23].

A large class of nonlinear systems can be represented by the well-known T-S multiple models due to their simplicity; using the interpolation between some linear submodels and their associated activation functions, any nonlinear system can be described by means of the T-S representation. Any nonlinear system can be transformed into a multiple model by using many approaches, the linearization method being the most used one; using the resulting interpolation of the 
linear models, a multiple model general form is obtained [9]:

$$
\begin{aligned}
& \dot{x}(t)=\sum_{i=1}^{M} \mu_{i}(\xi(t))\left[A_{i} x(t)+B_{i} u(t)+D v(t)+d_{i}\right], \\
& y(t)=C x(t),
\end{aligned}
$$

where $x(t) \in \mathscr{R}^{n}$ is the state vector, $u(t) \in \mathscr{R}^{m}$ the known input vector, $v(t) \in \mathscr{R}^{q}$ the unknown input vector, and $y(t) \in \mathscr{R}^{r}$ the measurable output vector, and $C \in \mathscr{R}^{r \times n}$, $D \in \mathscr{R}^{n \times q}$ and, for the local model "i", $A_{i} \in \mathscr{R}^{n \times n}, B_{i} \in$ $\mathscr{R}^{n \times m}$, and $d_{i} \in \mathscr{R}^{n}$ are considered known and constant matrices. The activation (weighting) functions $\mu_{i}(\xi(t)), i=$ $\overline{1, M}$, depending on the so-called decision vector $\xi(t)$, have the following properties [24]: $\sum_{i=1}^{M} \mu_{i}(\xi(t))=1 ; 0 \leq \mu_{i}(\xi(t)) \leq$ $1,(\forall) i=\overline{1, M} ; M$ is the number of local models depending on the precision of modeling, the complexity of the nonlinear system to be approximated by using a multiple model, and the structure of the activation functions. The matrices $A_{i}, B_{i}$, $C, D$, and $d_{i}$ can be obtained by the direct linearization of the nonlinear model around several operating points, or by means of an identification procedure [9].

Notations. Throughout the paper, the following notations are used: $X^{T}$ denotes the transpose of the matrix $X, X>0$ means that $X$ is a symmetric positive definite matrix, and $\|\cdot\|$ represents the Euclidean norm for vectors and the spectral norm for matrices, while $X^{*}$ is the right inverse of the full row rank matrix $X$, defined by the equation: $X^{*}=X^{T}\left(X X^{T}\right)^{-1}$ with the property: $X X^{*}=I$.

\section{Design of the New Reduced-Order Multiple Observer}

3.1. Problem Statement. The new reduced-order multiple observer is designed in this section for the case of T-S nonlinear systems with unknown inputs; after that, its robustness is improved by adding robustness terms in the observer's equations considering the case of uncertain multiple models. The direct and simple method to design the observer for T-S systems with unknown inputs reduces the design procedure of reduced-order observers with unknown inputs to the design problem of multiple observers. The new observer should estimate any state for Takagi-Sugeno multiple models with unknown inputs; the problem addressed in this paper is the design of a $p$-order reduced-order multiple observer which generates the required $p$ state vector of the form:

$$
z(t)=L x(t)
$$

where $L \in \mathscr{R}^{p \times n}$ is a full row rank matrix, while $z(t) \in \mathscr{R}^{p}$ is the vector containing a part of the system's state variables or combinations of these variables. The matrix $L$ has been chosen of the following form:

$$
L=T+F C,
$$

where $T \in \mathscr{R}^{p \times n}, F \in \mathscr{R}^{p \times r}$; the full row rank matrix $L$ is judiciously chosen and the matrices $T$ and $F$ have to be determined.
Without loss of generality, the next assumptions are considered in the paper: $(\mathrm{A} 1) \operatorname{rank}(C)=r, \operatorname{rank}(D)=q$; (A2) the pairs $\left(C, A_{i}\right)$ are observable; (A3) $p>r>q$; (A4) $n=p+r$. For matrix $C$ in (1) a particular form is chosen: $C=$ $\left\lfloor 0_{r \times(n-r)} \quad C_{2}\right\rfloor$, with $C_{2} \in \mathscr{R}^{r \times r}$ being full rank matrix $[25,26]$. This is not restrictive as long as the matrix $C$ is full row rank (condition (A1)), because there will always be an orthogonal transformation such that the output equation of the system is $y(t)=\left[\begin{array}{ll}0 & C_{2}\end{array}\right] x(t)$. It will be proved later that the only existence condition of ROMO is $\operatorname{rank}(C D)=\operatorname{rank}(D)=q$.

Consider $\widehat{z}(t)$ being the estimation of $z(t)$; the obtaining of this vector is achieved by using a $p$ order ROMO which has been obtained by modifying the form of the reduced-order functional observer from [20] and adapting it to the case of Takagi-Sugeno multiple models in [9]. The new ROMO can estimate the components of the state vector $x(t)$ by letting the matrix $L$ comprise those rows of identity matrix $I_{n}$ that correspond to the state variables to be estimated; having in mind that $L$ is a full row rank matrix, the estimated state vector $\widehat{x}(t)$ will be obtained using (2) and the right inverse of the matrix $L$; that is, $\widehat{x}(t)=L^{*} \widehat{z}(t)=L^{T}\left(L L^{T}\right)^{-1} \widehat{z}(t)$.

The reduced-order multiple observer for estimating the state vector of Takagi-Sugeno systems is chosen as follows:

$$
\begin{aligned}
\dot{w}(t) & =\sum_{i=1}^{M} \mu_{i}(\xi(t))\left[N_{i} w(t)+H_{i} u(t)+J_{i} y(t)+G_{i}\right], \\
\widehat{z}(t) & =w(t)+F y(t),
\end{aligned}
$$

with $w(t) \in \mathscr{R}^{p}$; to fully know the equations of the new ROMO, the matrices $N_{i} \in \mathscr{R}^{p \times p}, J_{i} \in \mathscr{R}^{p \times r}, H_{i} \in \mathscr{R}^{p \times m}$, $G_{i} \in \mathscr{R}^{p \times 1}, T \in \mathscr{R}^{p \times n}$, and $F \in \mathscr{R}^{p \times r}$ should be determined such that $\widehat{z}(t)$ converges to $z(t)$.

3.2. Global Convergence Conditions of the Reduced-Order Multiple Observer. For the determination of the new ROMO's unknown matrices, one considers the error vector of the observer $e(t)=z(t)-\widehat{z}(t)$. It is easy to prove that the dynamics of the new reduced-order multiple observer is

$$
\begin{aligned}
\dot{e}(t) & =\sum_{i=1}^{M} \mu_{i}(\xi(t)) N_{i} e+\sum_{i=1}^{M} \mu_{i}(\xi) \\
\cdot & {\left[\left(T A_{i}-N_{i} T-J_{i} C\right) x(t)+\left(T B_{i}-H_{i}\right) u(t)\right.} \\
+ & \left.T D v(t)+\left(T d_{i}-G_{i}\right)\right] .
\end{aligned}
$$

The convergence of the ROMO is synthesized into the following theorem.

Theorem 1. Consider the Takagi-Sugeno multiple model (1) with the assumptions (A1)-(A4). $e(t) \rightarrow 0$ as $t \rightarrow \infty$ for any $x(0), w(0), v(t)$, and $u(t)$ if and only if the matrix $\widetilde{N}=$ $\sum_{i=1}^{M} \mu_{i}(\xi) N_{i}$ is Hurwitz $\left(\sigma(\widetilde{N}) \in C_{-}\right)$and

$$
\begin{aligned}
T A_{i}-N_{i} T-J_{i} C & =0, \\
T B_{i}-N_{i} & =0, \\
T D & =0, \\
T d_{i}-G_{i} & =0 .
\end{aligned}
$$




\section{Proof.}

Sufficiency. If conditions (6) are satisfied, the error dynamics becomes $\dot{e}(t)=\widetilde{N} e(t)$; further, one gets $e(t)=e_{0} \cdot \exp (\widetilde{N} t)=$ $e(0) \cdot \exp (\widetilde{N} t)$ and, regardless of the value of $e_{0}$, if $\widetilde{N}$ is Hurwitz, $\lim _{t \rightarrow \infty} e(t)=0$ results for any $x(0), w(0), v(t)$, and $u(t)$.

Necessity. To proof the necessity of the theorem, the following property of propositions is used: Proposition $P \rightarrow$ propositions $P_{1} \Lambda P_{2} \Lambda P_{3} \Lambda P_{4} \Leftrightarrow \Gamma P_{1} \mathrm{~V} \Gamma P_{2} \mathrm{~V} \Gamma P_{3} \mathrm{~V} \Gamma P_{4} \rightarrow \Gamma P$ ( $\Lambda=$ and, $\mathrm{V}=$ or, $\Gamma=$ non). If $\widetilde{N}$ is not Hurwitz $\left(\sigma(\widetilde{N}) \notin C_{-}\right)$, even for $v(t)=0, u(t)=0, x(0)=0$ and $(\forall) e(0) \neq 0 \Rightarrow \dot{e}(0)=$ $\widetilde{N} e(0) \neq 0 \Rightarrow \lim _{t \rightarrow \infty} e(t) \neq 0$; if $T D \neq 0, T B_{i}-N_{i} \neq 0$, or $T d_{i}-G_{i} \neq 0$, then there exist $u(t)$ and $v(t)$ to make $\lim _{t \rightarrow \infty} e(t) \neq 0$; also, if $T A_{i}-N_{i} T-J_{i} C \neq 0$, because one supposed that there are no states which tend to zero for any values of the vectors $u(t)$ and $v(t), x(t) \neq 0$ results and the error dynamics is $\dot{e}(t)=\widetilde{N} e(t)+\left(T A_{i}-N_{i} T-J_{i} C\right) \underbrace{x(t)}_{\neq 0} \neq$

$\widetilde{N} e(t) \Rightarrow \lim _{t \rightarrow \infty} e(t) \neq 0$.

To determine other existence conditions of ROMO and to calculate the unknown matrices from its equations, one considers the Lyapunov function $V(e)=e^{T} P e$, where $P$ is a symmetrical and positive-defined matrix; using the dynamics of observer's error, one obtains the derivative of the function $V(e)$ as follows:

$$
\dot{V}(e)=\sum_{i=1}^{M} \mu_{i}(\xi)\left[e^{T}\left(N_{i}^{T} P+P N_{i}\right) e\right] .
$$

The convergence of the reduced-order multiple observer $(\dot{V}<$ 0 ) is achieved if the nonlinear matrix inequality

$$
N_{i}^{T} P+P N_{i}<0
$$

is satisfied (constraint of the observer) for any $i=\overline{1, M}$. Having in mind that, in the design algorithm of the ROMO (Section 5), the determination of the matrices $N_{i}$ will be achieved before solving the matrix inequalities (8), these will become linear matrix inequalities and will be easily solved by using the LMI theory.

To conclude, the new reduced-order multiple observer's design problem has been reduced now to the finding of the matrices $N_{i}, J_{i}, H_{i}, G_{i}, T$, and $P$ that satisfy the constraints:

$$
\begin{aligned}
& T A_{i}-N_{i} T-J_{i} C=0, \\
& T B_{i}-H_{i}=0, \\
& T D=0, \\
& T d_{i}-G_{i}=0, \\
& \sigma(\widetilde{N}) \in C_{-}, \\
& N_{i}^{T} P+P N_{i}<0 ;
\end{aligned}
$$

this problem is equivalent to the standard problem of observers' design when all the inputs are known.
3.3. Design Approach. The matrices $N_{i}, T, J_{i}, A_{i}, D$ are partitioned as

$$
\begin{aligned}
N_{i} & =\left[\begin{array}{ll}
N_{1}^{i} & N_{2}^{i} \\
N_{3}^{i} & N_{4}^{i}
\end{array}\right], \\
T & =\left[\begin{array}{ll}
T_{1} & T_{2} \\
T_{3} & T_{4}
\end{array}\right], \\
J_{i} & =\left[\begin{array}{l}
J_{1}^{i} \\
J_{2}^{i}
\end{array}\right], \\
A_{i} & =\left[\begin{array}{ll}
A_{1}^{i} & A_{2}^{i} \\
A_{3}^{i} & A_{4}^{i}
\end{array}\right], \\
D & =\left[\begin{array}{l}
D_{1} \\
D_{2}
\end{array}\right], \\
T_{3} & =\left[\begin{array}{ll}
T_{31} & T_{32}
\end{array}\right],
\end{aligned}
$$

with

$$
\begin{aligned}
& N_{i} \in \mathscr{R}^{p \times p}, \\
& N_{1}^{i} \in \mathscr{R}^{r \times r} \text {, } \\
& N_{2}^{i} \in \mathscr{R}^{r \times(p-r)} \text {, } \\
& N_{3}^{i} \in \mathscr{R}^{(p-r) \times r}, \\
& N_{4}^{i} \in \mathscr{R}^{(p-r) \times(p-r)}, \\
& T \in \mathscr{R}^{p \times n}, \\
& T_{1} \in \mathscr{R}^{r \times(n-r)} \text {, } \\
& T_{2} \in \mathscr{R}^{r \times r} \text {, } \\
& T_{3} \in \mathscr{R}^{(p-r) \times(n-r)}, \\
& T_{4} \in \mathscr{R}^{(p-r) \times r}, \\
& T_{31} \in \mathscr{R}^{(p-r) \times(n-p)}, \\
& T_{32} \in \mathscr{R}^{(p-r) \times(p-r)}, \\
& J_{i} \in \mathscr{R}^{p \times r}, \\
& J_{1}^{i} \in \mathscr{R}^{r \times r}, \\
& J_{2}^{i} \in \mathscr{R}^{(p-r) \times r}, \\
& D \in \mathscr{R}^{n \times q}, \\
& D_{1} \in \mathscr{R}^{p \times q} \text {, } \\
& D_{2} \in \mathscr{R}^{r \times q} \text {, } \\
& A_{i} \in \mathscr{R}^{n \times n} \text {, } \\
& A_{1}^{i} \in \mathscr{R}^{(n-r) \times(n-r)} \text {, }
\end{aligned}
$$




$$
\begin{aligned}
& A_{2}^{i} \in \mathscr{R}^{(n-r) \times r}, \\
& A_{3}^{i} \in \mathscr{R}^{r \times(n-r),} \\
& A_{4}^{i} \in \mathscr{R}^{r \times r} .
\end{aligned}
$$

The design of the new observer is concentrated into the following innovative theorem.

Theorem 2. Consider the Takagi-Sugeno multiple model (1); using assumptions (A1)-(A4) and the condition $\operatorname{rank}(C D)=$ $q$, the reduced-order multiple observer (4) is convergent if, for any $i=\overline{1, M}$, there exists a symmetrical and positive-defined matrix $P$ verifying the matrix inequality (8), if the matrix $\widetilde{N}=$ $\sum_{i=1}^{M} \mu_{i}(\xi) N_{i}$ is Hurwitz, and the matrices $T, G_{i}, H_{i}, F, N_{i}, J_{i}$ have the forms:

$$
\begin{aligned}
T & =I_{p \times n}-D_{1}(C D)^{+} C, \\
G_{i} & =T d_{i}, \\
H_{i} & =T B_{i}, \\
F & =\left(L-I_{p \times n}+D_{1}(C D)^{+} C\right)\left[\begin{array}{c}
0_{(n-r) \times r} \\
I_{r}
\end{array}\right] C_{2}^{-1}, \\
N_{i} & =\left[\begin{array}{ll}
N_{1}^{i} & \widetilde{A}_{1}^{i} \cdot\left[\begin{array}{c}
0_{(n-p) \times(p-r)} \\
I_{p-r}
\end{array}\right] \cdot T_{32}^{-1} \\
N_{3}^{i} & \widetilde{A}_{3}^{i} \cdot\left[\begin{array}{c}
0_{(n-p) \times(p-r)} \\
I_{p-r}
\end{array}\right] \cdot T_{32}^{-1}
\end{array}\right], \\
J_{i} & =\left[\begin{array}{ll}
\left(\widetilde{A}_{2}^{i}-\widetilde{A}_{1}^{i} \cdot\left[\begin{array}{c}
0_{(n-p) \times(p-r)} \\
I_{p-r}
\end{array}\right] \cdot T_{32}^{-1} \cdot T_{4}\right) C_{2}^{-1} \\
\left(\widetilde{A}_{4}^{i}-\widetilde{A}_{3}^{i} \cdot\left[\begin{array}{c}
0_{(n-p) \times(p-r)} \\
I_{p-r}
\end{array}\right] \cdot T_{32}^{-1} \cdot T_{4}\right) C_{2}^{-1}
\end{array}\right],
\end{aligned}
$$

where the matrices $N_{1}^{i}$ and $N_{3}^{i}$ have been randomly chosen, and

$$
\begin{aligned}
& \widetilde{A}_{1}^{i}=T_{1} A_{1}^{i}+T_{2} A_{3}^{i}-N_{1}^{i} T_{1}, \\
& \widetilde{A}_{2}^{i}=T_{1} A_{2}^{i}+T_{2} A_{4}^{i}-N_{1}^{i} T_{2}, \\
& \widetilde{A}_{3}^{i}=T_{3} A_{1}^{i}+T_{4} A_{3}^{i}-N_{3}^{i} T_{1}, \\
& \widetilde{A}_{4}^{i}=T_{3} A_{2}^{i}+T_{4} A_{4}^{i}-N_{3}^{i} T_{2} .
\end{aligned}
$$

Proof. The proof of Theorem 2 mainly involves the demonstration of the expressions (12), issue which is equivalent to the solving of the first four equations (9). The first equation to solve is the third equation (9), that is, $T D=0$; to determine the matrix $T$ from this equation, one chooses the matrix $T$ of the following form: $T=I_{p \times n}+E C$, with $I_{p \times n}$ being the identity matrix with $p$ lines and $n$ columns and $E \in \mathscr{R}^{p \times r}$ being an unknown matrix to be calculated. Using $C=\left\lfloor\begin{array}{lll}0_{r \times(n-r)} & C_{2}\end{array}\right\rfloor$ and partitioning the known matrix $D$ as in (10) and (11), one successively gets

$$
\begin{gathered}
\left(I_{p \times n}+E C\right) D=0 \Longleftrightarrow D_{1}+E C D=0 \Longleftrightarrow \\
E=-D_{1}(C D)^{+},
\end{gathered}
$$

where $(C D)^{+}$is the generalized pseudoinverse of $(C D)$, given by $[27,28]:(C D)^{+}=\left[(C D)^{T}(C D)\right]^{-1}(C D)^{T}$. The generalized pseudoinverse of $(C D)$ can be obtained if and only if $(C D)$ is full column rank, namely, $\operatorname{rank}(C D)=q$; till now, this is the only existence condition of the reduced-order multiple observer. With (14), one obtains the first expression (12), that is, $T=I_{p \times n}-D_{1}(C D)^{+} C$.

After the determination of the matrix $T$, by means of the second equation (9), $H_{i}=T B_{i}, i=\overline{1, M}$, results, and, by using the fourth equation (9), one gets $G_{i}=T d_{i}, i=\overline{1, M}$. The matrix $L$ is judiciously chosen by the designer of the observer having in mind the states which are directly measured by means of the system sensors (the forms of the matrices $C$ and $\mathrm{C}_{2}$ give this information) and the states that are not measured and should be estimated. The determination of the matrix $F$ is achieved now by using (3) and the obtained expression of the matrix $T$; thus, from (3) one obtains $F C=L-T \Leftrightarrow$ $F\left\lfloor 0_{r \times(n-r)} \quad C_{2}\right\rfloor=L-T$. By right multiplying of this equation with $\left[\begin{array}{c}0_{(n-r) \times r} \\ I_{r}\end{array}\right]$, taking into account the fact that $\operatorname{rank}(C)=$ $\operatorname{rank}\left(C_{2}\right)=r\left(C_{2}\right.$ is a full rank matrix $)$, one successively obtains $F=(L-T) \cdot\left[\begin{array}{c}0_{(n-r) \times r} \\ I_{r}\end{array}\right] \cdot C_{2}^{-1}=\left(L-I_{p \times n}+D_{1}(C D)^{+} C\right)$. $\left[\begin{array}{c}0_{(n-r) \times r} \\ I_{r}\end{array}\right] \cdot C_{2}^{-1}$. This way, the first four expressions (12) have been demonstrated.

The only unknown matrices from (4) of the ROMO are now $N_{i}$ and $J_{i}$; these are the solutions of the first equation (9) and subject of two constraints: $(1) \sigma(\widetilde{N}) \in C_{-}$; (2) for any $i=\overline{1, M}$, there exists a matrix $P>0$ which verifies the linear matrix inequality (8). One can use the method from [21] where the solving of an equation with two unknown matrices has been done in the design approach of a full-order observer for linear-time invariant systems with unknown inputs or the method from [25], where the matrices $N_{i}$ are calculated as

$$
N_{i}=T A_{i}-K_{i} C,
$$

where $K_{i}$ are obtained with the pole placement technique such that $\sigma\left(N_{i}\right) \in C_{-}$. Replacing (15) into the equation to be solved, that is, $T A_{i}-N_{i} T-J_{i} C=0$, and taking into account the equations $T=I_{p \times n}+E C, E=-D_{1}(C D)^{+}$, and $T=I_{p \times n}-D_{1}(C D)^{+} C$, the following is obtained:

$$
\begin{aligned}
& J_{i}=-T A_{i} E+K_{i}\left(I_{p \times n}+E C\right) \Longleftrightarrow \\
& J_{i}=T A_{i} D_{1}(C D)^{+}+K_{i}\left[I_{p \times n}-D_{1}(C D)^{+} C\right] ;
\end{aligned}
$$

to choose stable eigenvalues for the matrices $N_{i}$, the pair $\left(T A_{i}, C\right)$ should be observable [25]. If the pair $\left(T A_{i}, C\right)$ is not observable, the calculation of matrices $K_{i}$ is made such that the observer is asymptotically stable if and only if $\left(T A_{i}, C\right)$ 
is detectable. In [25], the equivalence of this condition with other two (see the next theorem) is proved.

Theorem 3 (see [25]). If conditions $\operatorname{rank}(D)=\operatorname{rank}(C D)=$ $q$ and $\operatorname{rank}(T)=n-q$ hold, the following 3 conditions are equivalent:

(1) the pair $\left(T A_{i}, C\right)$ is observable or at least detectable;

(2) $\operatorname{rank}\left[\underset{\mathrm{s}}{\mathrm{s} T-T A_{i}}\right]=n,(\forall) \mathbf{s} \in C, \operatorname{Re}\{\mathbf{s}\} \geq 0$;

(3) $\operatorname{rank}\left[\begin{array}{cc}\mathbf{s I}_{I_{n}}-A_{i} & D \\ C & 0\end{array}\right]=n+q,(\forall) \mathbf{s} \in C, \operatorname{Re}\{\mathbf{s}\} \geq 0$.

The main disadvantage of the approach presented in [25] is related to the choice of eigenvalues for the matrices $N_{i}$; the choice of the eigenvalues for the matrix $N_{i}$ has a great influence on the observer's poles and convergence speed. The pole placement technique is easy to implement in the case of observers' design, but it has some disadvantages: (1) the technique is difficult to be used in the case of systems with big order or for poorly controlled systems; (2) if one chooses fast poles for the observer, the advantage is that the observer estimation error decays rapidly, but the disadvantage is that the system needs perfect sensors and/or noise free environment; (3) if one chooses slow poles for the observer, the advantage is that the system is less sensitive to process disturbances and measurement noise, but the disadvantage is that the observer estimation error decays slowly [21].

The disadvantages of the pole placement technique lead to the conclusion that the approach from [21] is better than the one presented in [25]; therefore, one chooses to adapt the technique from [21] to the present case-multiple observer design for T-S multiple models with unknown inputs. This step firstly involves the partitioning of the matrices $N_{i}, T, J_{i}$, $A_{i}$ as in (10) and (11); substituting (10) into the first equation (9), one obtains

$$
\begin{aligned}
N_{1}^{i} T_{1}+N_{2}^{i} T_{3}-T_{1} A_{1}^{i}-T_{2} A_{3}^{i}=0_{r \times(n-r)}, \\
N_{1}^{i} T_{2}+N_{2}^{i} T_{4}+J_{1}^{i} C_{2}-T_{1} A_{2}^{i}-T_{2} A_{4}^{i}=0_{r \times r}, \\
N_{3}^{i} T_{1}+N_{4}^{i} T_{3}-T_{3} A_{1}^{i}-T_{4} A_{3}^{i}=0_{(p-r) \times(n-r)}, \\
N_{3}^{i} T_{2}+N_{4}^{i} T_{4}+J_{2}^{i} C_{2}-T_{3} A_{2}^{i}-T_{4} A_{4}^{i}=0_{(p-r) \times r} .
\end{aligned}
$$

The previous matrix equation system has 6 unknown variables (the matrices $N_{1}^{i}, N_{2}^{i}, N_{3}^{i}, N_{4}^{i}, J_{1}^{i}, J_{2}^{i}$ ) and only 4 equations. The system can be solved if only the number of unknown variables is equal to or less than the number of equations. Therefore, to transform the equation system (17) into a compatible determined system, two of the six matrices are considered random matrices. One chooses $N_{1}^{i} \in \mathscr{R}^{r \times r}$ and $N_{3}^{i} \in \mathscr{R}^{(p-r) \times r}$ randomly, but the approach also works well if, instead of these matrices, any other two matrices are chosen randomly. The other two matrices, together with $J_{1}^{i}$ and $J_{2}^{i}$, are now the 4 unknown variables of system (17) which becomes compatible determined, its solving being not a difficult task. Using the notations (13), system (17) gets the form:

$$
\begin{array}{r}
N_{2}^{i} T_{3}=\widetilde{A}_{1}^{i}, \\
N_{2}^{i} T_{4}+J_{1}^{i} C_{2}=\widetilde{A}_{2}^{i}, \\
N_{4}^{i} T_{3}=\widetilde{A}_{3}^{i}, \\
N_{4}^{i} T_{4}+J_{2}^{i} C_{2}=\widetilde{A}_{4}^{i} .
\end{array}
$$

By using the partitioning of matrix $T_{3}$ as in (10) and (11), the solving of the first equation (18) is equivalent with $N_{2}^{i}\left[\begin{array}{ll}T_{31} & T_{32}\end{array}\right]=\widetilde{A}_{1}^{i}$, which, by right multiplying with $\left[\begin{array}{c}0_{(n-p) \times(p-r)} \\ I_{p-r}\end{array}\right]$, leads to $N_{2}^{i}=\widetilde{A}_{1}^{i} \cdot\left[\begin{array}{c}0_{(n-p) \times(p-r)} \\ I_{p-r}\end{array}\right] \cdot T_{32}^{-1}$; in the same way, one solves the third equation (18) and gets $N_{4}^{i}=\widetilde{A}_{3}^{i}$. $\left[\begin{array}{c}{ }^{(n-p) \times(p-r)} \\ I_{p-r}\end{array}\right] \cdot T_{32}^{-1}$; one supposed that $T_{32}$ is a full rank matrix. The matrices $J_{1}^{i}$ and $J_{2}^{i}$ are determined from the second and the fourth equations of system (18); having again in mind that $C_{2}$ is a full rank matrix, the following is obtained:

$$
\begin{aligned}
J_{1}^{i} & =\left(\widetilde{A}_{2}^{i}-N_{2}^{i} T_{4}\right) C_{2}^{-1} \\
& =\left(\widetilde{A}_{2}^{i}-\widetilde{A}_{1}^{i} \cdot\left[\begin{array}{c}
0_{(n-p) \times(p-r)} \\
I_{p-r}
\end{array}\right] \cdot T_{32}^{-1} \cdot T_{4}\right) C_{2}^{-1}, \\
J_{2}^{i} & =\left(\widetilde{A}_{4}^{i}-N_{4}^{i} T_{4}\right) C_{2}^{-1} \\
& =\left(\widetilde{A}_{4}^{i}-\widetilde{A}_{3}^{i} \cdot\left[\begin{array}{c}
0_{(n-p) \times(p-r)} \\
I_{p-r}
\end{array}\right] \cdot T_{32}^{-1} \cdot T_{4}\right) C_{2}^{-1} ;
\end{aligned}
$$

the last two expressions (12) have been demonstrated.

The determined matrices $N_{i}$ should satisfy two constraints: $(\mathrm{C} 1) \sigma(\widetilde{N}) \in C_{-} ;(\mathrm{C} 2)$ for any $i=\overline{1, M}$, there exists a matrix $P>0$ which verifies the matrix inequality (8). Using the matrices $N_{i}, i=\overline{1, M}$, the matrix $\widetilde{N}$ and its eigenvalues are obtained; also, the $M$ linear matrix inequalities (8) are solved; if the matrices $N_{i}, i=\overline{1, M}$, assure the stability of the matrix $\widetilde{N}$ (all its eigenvalues are placed in the left-hand side of the complex plane) and the existence of a matrix $P>0$ such that, for any $i=\overline{1, M}$, the LMIs (8) are satisfied, the matrices $N_{i}$ have been obtained properly, the dynamics of the observer error has a homogeneous form and, according to Theorem 1, $e(t) \rightarrow 0$ as $t \rightarrow \infty$ for any $x(0), w(0), v(t)$, and $u(t)$. Otherwise, other random matrices $N_{1}^{i}$ and $N_{3}^{i}$ are chosen and system (18) is again solved until the constraints (C1) and (C2) are fulfilled. In the new algorithm software implementation, the fulfillment of the two constraints is made in a "while" loop. To conclude, one managed to avoid the pole placement technique; thus, no condition regarding the observability or detectability of the pair $\left(T A_{i}, C\right)$ is needed in our design approach and, therefore, the only existence condition of the observer is $\operatorname{rank}(C D)=\operatorname{rank}(D)=q$. As a consequence, the introduced innovation element (the "while" loop) increases the generality and convergence of the new ROMO. The theorem's proof is now complete. 


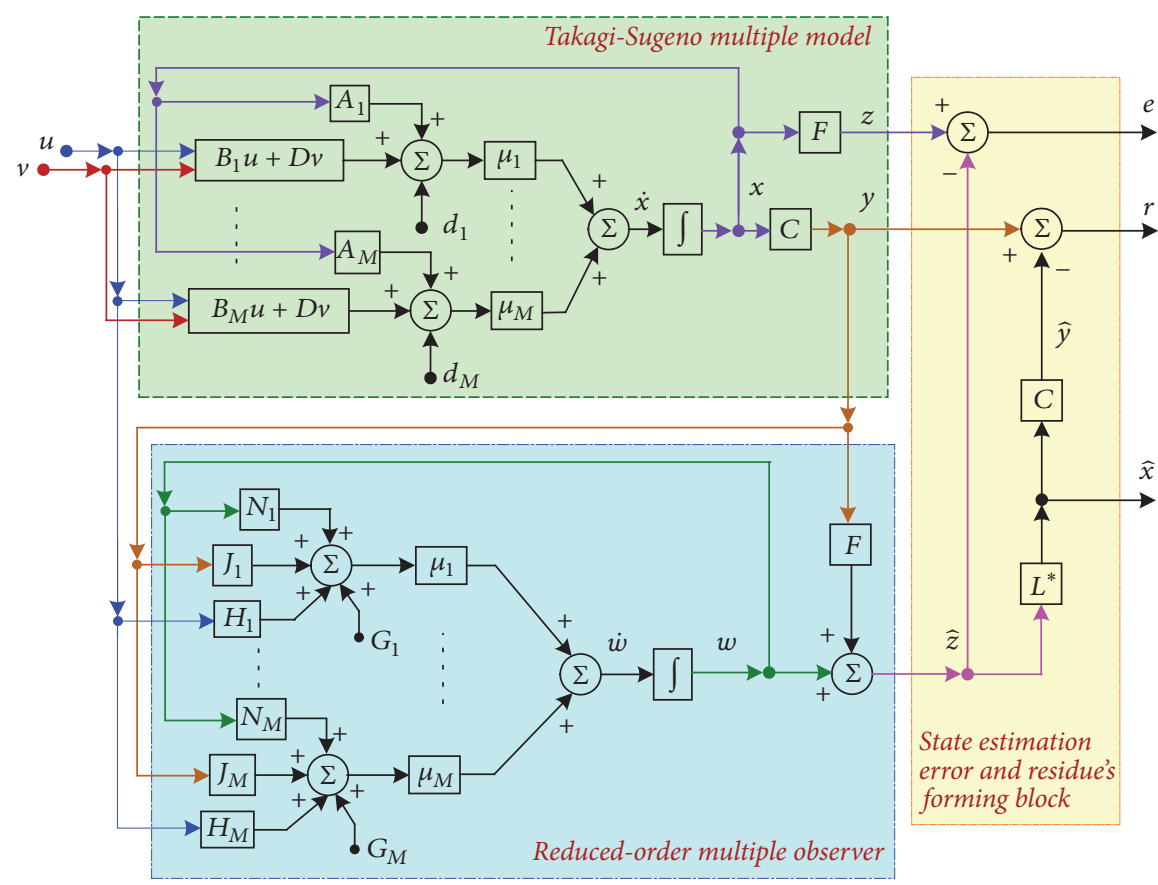

FIGURE 1: Block diagram of the reduced-order multiple observer.

Remark 4. It is well known that the information provided by the sensors of a system is in connection with the output matrix of the system $(C)$ and vice versa; if one chooses to measure the last $r$ states from the state vector $x$, the matrix $C$ has the form $C=\left\lfloor\begin{array}{ll}0_{r \times(n-r)} & I_{r}\end{array}\right\rfloor$; in this case, the only existence condition of the reduced-order multiple observer is always satisfied and, therefore, the new algorithm has no existence conditions and only 4 assumptions have to be made. Thus, another important advantage of the new ROMO is the lack of a priori restrictions on the class of Takagi-Sugeno multiple models that can be considered.

The block diagram associated with the ensemble multiple model-reduced-order multiple observer-is presented in Figure 1.

\section{Improvement of the Observer's Robustness}

The matrices $A_{i}$ from (1) may be not completely known; in this case, some small unknown variances $\left(\Delta A_{i}\right)$ can appear, the multiple model being affected by uncertainties (modeling unknown parameters). Therefore, the improvement of the ROMO's robustness is a problem to solve from the unknown small variances' point of view such that the negative effect of the unknown matrices on the system state estimation is minimized, if not canceled. The apparition of the uncertain matrices $\Delta A_{i}$ in the dynamics of the system is the negative consequence of faults, calculation errors, modeling errors, or small variations of the system during the linearization process [29]. If the uncertain matrices are taken into consideration, the multiple model, initially described by (1), becomes now

$$
\dot{x}(t)=\sum_{i=1}^{M} \mu_{i}(\xi(t))
$$

$$
\begin{aligned}
& \cdot\left[\left(A_{i}+\Delta A_{i}\right) x(t)+B_{i} u(t)+D v(t)+d_{i}\right], \\
& y(t)=C x(t) ;
\end{aligned}
$$

if $\Delta A_{i}=0,(1)$ are obtained; otherwise, the effect of the unknown matrices $\Delta A_{i}$ cannot be completely eliminated but only minimized. To overcome the drawback related to the presence of the uncertain matrices (considered unknown terms) and to improve the robustness of the ROMO with respect to these uncertainties, the robustness (compensation) terms $\gamma_{i}(t), i=\overline{1, M}$, are added in the equations of the observer; its new form is

$$
\begin{aligned}
& \dot{w}(t) \\
& =\sum_{i=1}^{M} \mu_{i}(\xi(t))\left[N_{i} w(t)+H_{i} u(t)+J_{i} y(t)+G_{i}+\gamma_{i}\right], \\
& \widehat{z}(t)=w(t)+F y(t) .
\end{aligned}
$$

The determination of the terms $\gamma_{i}(t)$ is achieved by using the Lyapunov theory; this approach represents another innovative element of the paper. The expression of the robustness terms is presented within the following theorem.

Theorem 5. For any $i=\overline{1, M}$, if there exist a matrix $\bar{P}>0$ and the positive scalars $\beta_{1}, \beta_{2}$, satisfying the constraint

$$
\left[\begin{array}{cc}
N_{i}^{T} \bar{P}+\bar{P} N_{i}+\beta_{1} \widetilde{T}_{i}^{A}+\beta_{1} \beta_{2}^{-1} \widetilde{\delta}_{i}^{A} I & \bar{P} \\
\bar{P} & -\beta_{1}^{-1} I
\end{array}\right]<0,
$$


the state estimation error of the observer (21) converges to zero if

$$
\begin{aligned}
& \gamma_{i}(t) \\
& = \begin{cases}\frac{\beta_{1}\left(1+\beta_{2}\right) \widetilde{\delta}_{i}^{A} \widehat{z}(t)^{T} \widehat{z}(t)}{2} \cdot \widetilde{P} \frac{r(t)}{r^{T}(t) r(t)}, & r(t) \neq 0, \\
0, & r(t)=0,\end{cases}
\end{aligned}
$$

where $r(t)=y(t)-\widehat{y}(t), \widetilde{P}=\bar{P}^{-1}\left[\left(L C^{*}\right)^{+}\right]^{T}$, and the time variant matrices $\widetilde{T}_{i}^{A}=\left(L^{*}\right)^{T}\left(T \Delta A_{i}\right)^{T}\left(T \Delta A_{i}\right) L^{*}$ are bounded, that is, $\left\|\widetilde{T}_{i}^{A}\right\|<\widetilde{\delta}_{i}^{A}$.

Proof. To deduce the expression of the robustness terms $\gamma_{i}(t), i=\overline{1, M}$, one calculates again the dynamics of the ROMO's error $\dot{e}(t)=\dot{z}(t)-\dot{\vec{z}}(t)=T \dot{x}(t)-\dot{w}(t)$ and, by using the previous equations and $w(t)=T x(t)-e(t)$, the following is obtained:

$$
\dot{e}(t)=\sum_{i=1}^{M} \mu_{i}(\xi)\left[N_{i} e(t)-T_{i}^{A} x(t)-\gamma_{i}(t)\right],
$$

where $T_{i}^{A}=T \cdot \Delta A_{i}$; to obtain equation (24), the fulfillment of the constraints from Theorem 2 has been considered. For the ease of notations, in the below presented approach, the time dependence $(t)$ is omitted.

Using the Lyapunov function $V(e)=e^{T} \bar{P} e$, with $\bar{P}$ being symmetrical and positive-defined matrix and the dynamics of observer's error being (24), one obtains the derivative of the function $V(e)$ as follows:

$$
\begin{aligned}
\dot{V}= & \dot{e}^{T} P e+e^{T} P \dot{e} \\
= & \sum_{i=1}^{M} \mu_{i}(\xi) e^{T}\left(N_{i}^{T} \bar{P}+\bar{P} N_{i}\right) e \\
& +\sum_{i=1}^{M} \mu_{i}(\xi)\left(x^{T} T_{i}^{A^{T}} \bar{P} e+e^{T} \bar{P} T_{i}^{A} x-2 \gamma_{i}^{T} \bar{P} e\right)
\end{aligned}
$$

the equation $\gamma_{i}^{T} \bar{P} e+e^{T} \bar{P} \gamma_{i}=2 \gamma_{i}^{T} \bar{P} e$ has been used.

During the proof of this theorem, the following inequality is used [8]:

$$
X^{T} Y+Y^{T} X \leq \beta X^{T} X+\beta^{-1} Y^{T} Y,
$$

for any matrices $X$ and $Y$ and positive scalars $\beta$.

Using inequality (26), written for the set: $\left(\Sigma_{1}\right):\{X=$ $\left.T_{i}^{A} x, Y=\bar{P} e, \beta=\beta_{1}\right\}$, the following is obtained: $x^{T} T_{i}^{A^{T}} \bar{P} e+$ $e^{T} \bar{P} T_{i}^{A} x \leq \beta_{1} x^{T} \bar{T}_{i}^{A} x+\beta_{1}^{-1} e^{T} \bar{P}^{2} e$, with $\bar{T}_{i}^{A}=T_{i}^{A^{T}} \cdot T_{i}^{A}$. By using these, (25) becomes

$$
\begin{aligned}
\dot{V} \leq & \sum_{i=1}^{M} \mu_{i}(\xi)\left[e^{T}\left(N_{i}^{T} \bar{P}+\bar{P} N_{i}+\beta_{1}^{-1} \bar{P}^{2}\right) e\right] \\
& +\sum_{i=1}^{M} \mu_{i}(\xi)\left(\beta_{1} x^{T} \bar{T}_{i}^{A} x-2 \gamma_{i}^{T} \bar{P} e\right) .
\end{aligned}
$$

Using the expression of the error $e=z-\widehat{z}=L x-\widehat{z} \Leftrightarrow$ $x=L^{*}(e+\widehat{z})$, the term $\beta_{1} x^{T} \bar{T}_{i}^{A} x$ can be expressed as follows:

$$
\begin{aligned}
\beta_{1} x^{T} \bar{T}_{i}^{A} x= & \beta_{1} \widehat{z}^{T} \widetilde{T}_{i}^{A} \widehat{z}+\beta_{1} e^{T} \widetilde{T}_{i}^{A} e \\
& +\beta_{1}\left(\widehat{z}^{T} \widetilde{T}_{i}^{A} e+e^{T} \widetilde{T}_{i}^{A} \widehat{z}\right),
\end{aligned}
$$

with $\widetilde{T}_{i}^{A}=\left(L^{*}\right)^{T}\left(T \Delta A_{i}\right)^{T}\left(T \Delta A_{i}\right) L^{*}=$ $\left[L^{T}\left(L L^{T}\right)^{-1}\right]^{T}\left(T \Delta A_{i}\right)^{T}\left(T \Delta A_{i}\right)\left[L^{T}\left(L L^{T}\right)^{-1}\right]$. Also, considering that the matrices $\widetilde{T}_{i}^{A}$ are bounded, that is, $\left\|\widetilde{T}_{i}^{A}\right\|<\widetilde{\delta}_{i}^{A}$, using again inequality (26) for the set $\left(\Sigma_{2}\right):\{X=\widehat{z}, Y=e, \beta=$ $\left.\beta_{2}\right\}$, inequality (27) gets the form:

$$
\begin{aligned}
\dot{V} & \leq \sum_{i=1}^{M} \mu_{i}(\xi) \\
& \cdot\left[e^{T}\left(N_{i}^{T} \bar{P}+\bar{P} N_{i}+\beta_{1}^{-1} \bar{P}^{2}+\beta_{1} \widetilde{T}_{i}^{A}+\beta_{1} \beta_{2}^{-1} \widetilde{\delta}_{i}^{A} I\right)\right. \\
& \cdot e]+\sum_{i=1}^{M} \mu_{i}(\xi)\left[\beta_{1}\left(1+\beta_{2}\right) \widetilde{\delta}_{i}^{A} \widehat{z}^{T} \widehat{z}-2 \gamma_{i}^{T} \bar{P} e\right]
\end{aligned}
$$

Imposing $\beta_{1}\left(1+\beta_{2}\right) \widetilde{\delta}_{i}^{A} \widehat{z}^{T} \widehat{z}-2 \gamma_{i}^{T} \bar{P} e=0$, (29) becomes $\dot{V} \leq$ $\sum_{i=1}^{M} \mu_{i}(\xi)\left[e^{T}\left(N_{i}^{T} \bar{P}+\bar{P} N_{i}+\beta_{1}^{-1} \bar{P}^{2}+\beta_{1} \widetilde{T}_{i}^{A}+\beta_{1} \beta_{2}^{-1} \widetilde{\delta}_{i}^{A} I\right) e\right] ;$ the convergence of the ROMO for uncertain Takagi-Sugeno systems with unknown inputs is achieved if, for any $i=$ $\overline{1, M}$, there exists a symmetrical and positive-defined matrix $\bar{P}$ which verifies the nonlinear matrix inequality:

$$
N_{i}^{\mathrm{T}} \bar{P}+\bar{P} N_{i}+\beta_{1}^{-1} \bar{P}^{2}+\beta_{1} \widetilde{T}_{i}^{A}+\beta_{1} \beta_{2}^{-1} \widetilde{\delta}_{i}^{A} I<0,
$$

which, by using the Schur lemma, is equivalent to the linear matrix inequality (22).

The obtaining of (23) is achieved by means of the relationship $\beta_{1}\left(1+\beta_{2}\right) \widetilde{\delta}_{i}^{A} \widehat{z}^{T} \widehat{z}=2 \gamma_{i}^{T} \bar{P} e$; from this, one gets $\gamma_{i}=\left[\left(\beta_{1}\left(1+\beta_{2}\right) \widetilde{\delta}_{i}^{A} \widehat{z}^{T} \widehat{z} / 2\right) e^{+} \bar{P}^{-1}\right]^{T}=\beta_{1}\left(1+\beta_{2}\right) \widetilde{\delta}_{i}^{A} \widehat{z}^{T} \widehat{z} / 2$. $\left(e^{+} \bar{P}^{-1}\right)^{T}$. Using the equations (1) $\bar{P}=\bar{P}^{T}$; (2) $e=L(x-$ $\widehat{x})=L C^{*}(y-\widehat{y})=L C^{*} r$, with $r=y-\widehat{y}$ (residue of the ROMO); (3) $\left(Z^{+}\right)^{T}=\left[\left(Z^{T} Z\right)^{-1} Z^{T}\right]^{T}=\left(Z^{T}\right)^{T}\left[\left(Z^{T} Z\right)^{-1}\right]^{T}=$ $\left(Z^{T}\right)^{T}\left(Z^{T} Z\right)^{-1}=\left(Z^{T}\right)^{*}$, for any column vector $Z$; $(4)\left(r^{+}\right)^{T}=$ $\left(r^{T}\right)^{*}=r\left(r^{T} r\right)^{-1}=r / r^{T} r$, one calculates $\left(e^{+} \bar{P}^{-1}\right)^{T}$ as follows:

$$
\begin{aligned}
\left(e^{+} \bar{P}^{-1}\right)^{T} & =\bar{P}^{-1}\left(e^{+}\right)^{T}=\bar{P}^{-1}\left[\left(L C^{*} r\right)^{+}\right]^{T} \\
& =\bar{P}^{-1}\left[r^{+}\left(L C^{*}\right)^{+}\right]^{T}=\bar{P}^{-1}\left[\left(L C^{*}\right)^{+}\right]^{T} \frac{r}{r^{T} r} \\
& =\widetilde{P} \frac{r}{r^{T} r},
\end{aligned}
$$

where $\widetilde{P}=\bar{P}^{-1}\left[\left(L C^{*}\right)^{+}\right]^{T}=\bar{P}^{-1}\left[\left(L C^{T}\left(C C^{T}\right)^{-1}\right)^{+}\right]^{T}$.

With this, the expression of the robustness terms becomes

$$
\gamma_{i}=\frac{\beta_{1}\left(1+\beta_{2}\right) \widetilde{\delta}_{i}^{A} \widehat{z}^{T} \widehat{z}}{2} \cdot \widetilde{P} \cdot \frac{r}{r^{T} r} .
$$

A problem which can appear in the ROMO's implementation process is related to the case when, although $r \rightarrow 0$, 
the robustness terms $\gamma_{i}$ are not bounded. For this problem, the solution from [8] can be borrowed: $\gamma_{i}$ are null if $\|r\|<\varepsilon$, where $\varepsilon$ is a positive constant having small values, chosen by the observer designer. In this case, the observer estimation error does not converge asymptotically to zero but very close according to the values of $\varepsilon$. Thus, the expression of the robustness terms (23) has been deduced and the proof of the theorem is now complete.

\section{Design Algorithm for Software Implementation}

The ROMO's design procedure, presented in detail in Sections 3 and 4, can be summarized now into the following algorithm.

Step 1. One introduces the matrices/vectors $A_{i}, B_{i}, d_{i}, i=$ $\overline{1, M}$, and the constant matrices $C, D ; r=\operatorname{rank}(C)=$ $\operatorname{rank}\left(C_{2}\right)$ and $p=n-r$ ( $n$ is the number of states) are calculated; one checks the fulfillment of the ROMO's assumptions $((\mathrm{A} 1)-(\mathrm{A} 4))$ and existence condition: $\operatorname{rank}(C D)=q$; the expressions of the decision vector $\xi(t)$ and activation functions $\mu_{i}(\xi(t)), i=\overline{1, M}$, are established.

Step 2. One judiciously chooses the full row rank matrix $L$.

Step 3. The matrices $N_{i}, T, J_{i}, A_{i}, D$ are partitioned with (10) and (11); the matrix $D_{1}$ is obtained by using (11), while the matrices $T, G_{i}, H_{i}$, and $F$ are calculated by means of first four equations (12).

Step 4. One randomly chooses the matrices $N_{1}^{i} \in \mathscr{R}^{r \times r}$ and $N_{3}^{i} \in \mathscr{R}^{(p-r) \times r}, \quad i=\overline{1, M}$, and the matrices (13) are calculated. Using these matrices and the last two equations (12), the last four unknown matrices $\left(N_{2}^{i}, N_{4}^{i}, J_{1}^{i}, J_{2}^{i}\right)$ are obtained.

Step 5. The matrices $N_{i}$ and $J_{i}, i=\overline{1, M}$, are built, and the matrix $\widetilde{N}=\sum_{i=1}^{M} \mu_{i}(\xi) N_{i}$ and its eigenvalues are calculated. For any $i=\overline{1, M}$, the LMIs (8) are solved with respect to $P$; if the constraints $(\mathrm{C} 1)$ and $(\mathrm{C} 2)$ are satisfied, the matrices $N_{i}$ and $J_{i}$ have been obtained properly; otherwise one returns to Step 4 and repeats Steps 4 and 5 until the two conditions are fulfilled.

Step 6. By means of the calculated matrices, one designs the observer described by (4) - the case without uncertainties; the time histories of the states and estimated states are obtained; using these vectors, the residual $r$ is calculated. If the condition $\|r\|<\varepsilon$ is not fulfilled, the positive scalars $\beta_{1}$, $\beta_{2}$ are chosen and the bounds $\widetilde{\delta}_{i}^{A}$ are calculated; the LMIs (22) are solved with respect to matrix $\bar{P}$; then, one calculates the matrix $\widetilde{P}$ and the robustness terms $\gamma_{i}(t), i=\overline{1, M}$, by means of (23); these additional terms are introduced in the equations of the observer for the case of dynamics with uncertainties. For the validation of the reduced-order multiple observer, the dynamics associated with the multiple model ((1): case without uncertainties or (20): case with uncertainties) is also used.

\section{Numerical Simulation Results}

6.1. Numerical Simulation Setup. To validate the new reduced-order observer, any dynamics meeting the assumptions and the existence condition can be chosen; in this paper, the new ROMO is software implemented and validated in Matlab for the case of a light aircraft lateral-directional motion; the aircraft dynamics, with $M=2$, has the form (1) where the following matrices have been considered [24]:

$$
\begin{aligned}
& A_{1} \\
& =\left[\begin{array}{ccccccc}
-0.3 & 0 & -33 & 9.81 & 0 & -5.4 & 0 \\
0.1 & -8.3 & 3.75 & 0 & 0 & 0 & -28.6 \\
0.37 & 0 & -0.64 & 0 & 0 & -9.5 & 0 \\
0 & 1 & 0.01 & 0 & 0.01 & 0 & 0 \\
0 & 0 & 1 & 0.001 & 0 & 0.001 & 0 \\
0 & 0 & -0.01 & 0 & 0 & -10 & 0 \\
0 & 0 & 0.01 & 0 & -0.001 & 0 & -5
\end{array}\right],
\end{aligned}
$$

$A_{2}$

$$
=\left[\begin{array}{ccccccc}
-0.28 & 0 & -33 & 9.81 & 0 & -5.2 & 0 \\
0.1 & -7.6 & 3.95 & 0 & 0 & 0 & -27.6 \\
0.34 & 0 & -0.59 & 0 & 0 & -9.6 & 0 \\
0 & 1 & 0.01 & 0 & 0.01 & 0 & 0 \\
0 & 0 & 1 & 0.002 & 0 & 0.001 & 0 \\
0 & 0 & -0.02 & 0 & 0 & -9.9 & 0 \\
0 & 0 & 0.02 & 0 & -0.001 & 0 & -4.95
\end{array}\right] \text {, }
$$

$B_{1}=\left[\begin{array}{cc}0 & 0 \\ 0 & 0 \\ 0 & 0 \\ 0 & 0 \\ 0 & -0.012 \\ 10 & 0 \\ 0 & 10\end{array}\right]$

$B_{2}=\left[\begin{array}{cc}0 & 0 \\ 0 & 0 \\ 0 & 0 \\ 0 & 0 \\ 0 & -0.009 \\ 9.8 & 0 \\ 0 & 9.8\end{array}\right]$

$d_{1}=0.2 \cdot\left[\begin{array}{l}1 \\ 1 \\ 1 \\ 1 \\ 1 \\ 1 \\ 1\end{array}\right]$ 


$$
\begin{aligned}
& d_{2}=0.1 \cdot\left[\begin{array}{l}
1 \\
1 \\
1 \\
1 \\
1 \\
1 \\
1
\end{array}\right] \text {, } \\
& C=\left[\begin{array}{ll}
C_{1} & C_{2}
\end{array}\right]=\left[\begin{array}{ll}
0_{3 \times 4} & I_{3}
\end{array}\right] \text {; } \\
& D=\left[\begin{array}{lllllll}
0.5 & 0 & 0.5 & 1 & 0 & 2 & 0
\end{array}\right]^{T} \text {. }
\end{aligned}
$$

The state of the system is $x=\left[\begin{array}{lllllll}V_{y} & \omega_{x} & \omega_{z} & \varphi & \psi & \delta_{r} & \delta_{a}\end{array}\right]^{T}$, with $V_{y}$ being aircraft lateral velocity, $\omega_{x}$ the roll angular rate, $\omega_{z}$ the yaw angular rate, $\varphi$ the roll angle of aircraft, $\psi$ the yaw angle of aircraft, $\delta_{r}$ the rudder deflection, and $\delta_{a}$ the ailerons' deflection; the two components of the system input vector $u=\left[\begin{array}{ll}\delta_{r_{c}} & \delta_{a_{c}}\end{array}\right]^{T}$ are the commands of the rudder and ailerons, respectively [24].

Aircraft flight is often influenced by disturbances like wind shears, atmospheric turbulences, or errors of the sensors. From aircraft dynamics' point of view, these represent unknown inputs; an observer for systems with unknown inputs may estimate these unknown inputs, but, more important, it must estimate the system states with very small errors [4]; therefore, in this paper, the unknown input vector $v(t)$ has been randomly chosen. The decision and the activation functions have been chosen of the following forms: $\xi(t)=$ $u(t), \mu_{1}(\xi(t))=0.4(1-\tanh (\xi(t))), \mu_{2}(\xi(t))=1-\mu_{1}(\xi(t))$, where the input has the form $u=-\bar{K} \hat{x}$, with the gain matrix $\bar{K}$ determined by using the ALGLX optimal algorithm borrowed from [24]; $\bar{K}$ can be also calculated by means of the pole placement technique or other methods.

6.2. Results and Discussion. For the above presented matrices, one obtains $n=7, m=2, q=1$, and $p=4$. The observer validation is performed for both the dynamics without uncertainties $\left(\Delta A_{i}=0\right)$ and with uncertainties $\left(\Delta A_{i} \neq 0\right)$. The solving of the linear matrix inequalities (8) and (22) is performed using the Matlab LMI tool. From the forms of the matrices $C$ and $C_{2}$, one can remark that the last three states of the system $\left(\psi, \delta_{r}, \delta_{a}\right)$ are measured; within Step 2 of the new algorithm, one judiciously chooses the full row rank matrix $L$; since this matrix gives information regarding the estimated states, one chooses $L=\left\lfloor\begin{array}{ll}I_{p} & 0_{p \times(n-p)}\end{array}\right]$; thus, the first $p=4$ states are estimated, while the last 3 are measured. Having in mind the form of the matrix $C$, one gets $y(t)=\left[\begin{array}{ll}0_{r \times(n-r)} & I_{r}\end{array}\right] x(t)=\left[\begin{array}{ll}0_{r \times(n-r)} & I_{r}\end{array}\right]\left[\begin{array}{l}x_{1}(t) \\ x_{2}(t)\end{array}\right]=x_{1}(t)$; in other words, $x_{1}(t)$ contains the measurable states, while $z(t)=\left[\begin{array}{ll}I_{p} & 0_{p \times(n-p)}\end{array}\right] x(t)=\left[\begin{array}{ll}I_{p} & 0_{p \times(n-p)}\end{array}\right]\left[\begin{array}{l}x_{1}(t) \\ x_{2}(t)\end{array}\right]=x_{2}(t)$ contains the states to be estimated (the last $r$ states of the system). Thus, ROMO estimates $x_{2}(t)$, providing $\widehat{x}_{2}(t)$; the estimate of the state vector $x(t)$ is obtained by concatenation of the vectors $x_{1}(t)$ and $\widehat{x}_{2}(t)$; that is, $\widehat{x}(t)=\left[\begin{array}{l}x_{1}(t) \\ \widehat{x}_{2}(t)\end{array}\right]=\left[\begin{array}{l}y(t) \\ \widehat{z}(t)\end{array}\right]$.
For the first simulation case (without uncertainties), within Steps 3, 4, and 5, one obtains

$$
\begin{aligned}
T & =\left[\begin{array}{lllllcl}
1 & 0 & 0 & 0 & 0 & -0.25 & 0 \\
0 & 1 & 0 & 0 & 0 & 0 & 0 \\
0 & 0 & 1 & 0 & 0 & -0.25 & 0 \\
0 & 0 & 0 & 1 & 0 & -0.5 & 0
\end{array}\right], \\
G_{1} & =\left[\begin{array}{c}
0.015 \\
0.02 \\
0.015 \\
0.01
\end{array}\right],
\end{aligned}
$$$$
G_{2}=\left[\begin{array}{c}
0.0075 \\
0.01 \\
0.0075 \\
0.005
\end{array}\right] \text {, }
$$$$
H_{1}=\left[\begin{array}{cc}
-2.5 & 0 \\
0 & 0 \\
-2.5 & 0 \\
-5 & 0
\end{array}\right],
$$$$
H_{2}=\left[\begin{array}{cc}
-2.45 & 0 \\
0 & 0 \\
-2.45 & 0 \\
-4.9 & 0
\end{array}\right] \text {, }
$$

$$
F=\left[\begin{array}{ccc}
0 & 0.25 & 0 \\
0 & 0 & 0 \\
0 & 0.25 & 0 \\
0 & 0.5 & 0
\end{array}\right] \text {, }
$$

$$
\begin{aligned}
& N_{1}=\operatorname{diag}[-5 ;-10 ;-15 ;-20], \\
& N_{2}=\operatorname{diag}[-7.5 ;-15 ;-22.5 ;-30], \\
& J_{1}=\left[\begin{array}{ccc}
0 & -4.27 & 0 \\
0 & 0 & -29.76 \\
0 & -11.03 & 0 \\
0.01 & -4.8 & 0
\end{array}\right], \\
& J_{2}=\left[\begin{array}{ccc}
0 & -4.26 & 0 \\
0 & 0 & -29.77 \\
0 & -11.04 & 0 \\
0.01 & -4.79 & 0
\end{array}\right] ;
\end{aligned}
$$

the solutions of the two LMIs $(8)$ are $P_{1}=\operatorname{diag}[0.195 ; 0.105$; $0.06 ; 0.04], P_{2}=\operatorname{diag}[0.072 ; 0.035 ; 0.023 ; 0.017]$.

For increasing the robustness of the ROMO, one uses the new technique involving the adding of $M$ robustness 


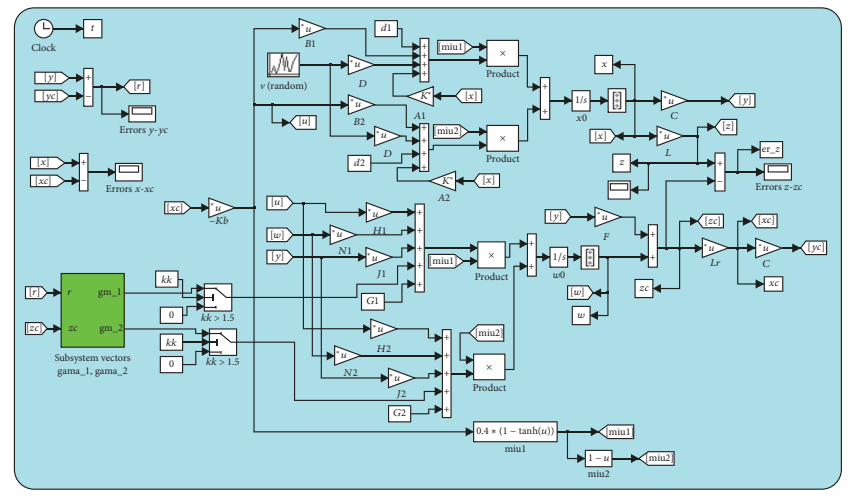

(a)

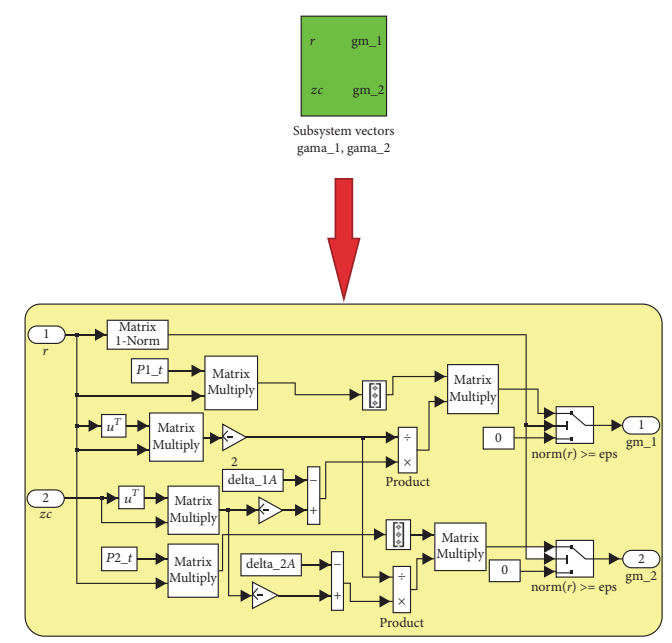

(b)

FIGURE 2: Matlab/Simulink model for the new reduced-order multiple observer.

terms in the observer's equations; thus, for case 2 (dynamics with uncertainties), one has chosen $\Delta A_{i}$ as a percent of $1 \%$ from their initial values $\left(A_{i}\right)$; also, one has chosen $\varepsilon=$ 0.01 and different values for the scalars $\beta_{1}$ and $\beta_{2}\left(\beta_{1}=\right.$ $\left.\beta_{2}=0.8 ; 1.6 ; 2.4 ; 3.2\right)$ in order to analyze the influence of these constants on the observer's convergence time and convergence errors. Solving the two linear matrix inequalities (22) for $\beta_{1}=\beta_{2}=0.8$, the following is obtained:

$$
\begin{aligned}
& \bar{P}_{1}=10^{-2} \cdot\left[\begin{array}{cccc}
13.53 & 0 & 0 & 0 \\
0 & 6.65 & -0.01 & 0 \\
0 & -0.01 & 4.66 & -0.07 \\
0 & 0 & -0.07 & 3.27
\end{array}\right], \\
& \bar{P}_{2}=10^{-2} \cdot\left[\begin{array}{cccc}
8.82 & 0 & 0 & 0 \\
0 & 4.34 & -0.01 & 0 \\
0 & -0.01 & 3.05 & -0.05 \\
0 & 0 & -0.05 & 2.15
\end{array}\right] .
\end{aligned}
$$

The new architecture from Figure 1 is software implemented in Matlab, Figure 2(a); the Matlab/Simulink subsystem "subsystem vectors gama_1, gama_2" (subsystem for the calculation of the vectors $\left.\gamma_{i}(t), i=\overline{1, M}\right)$ is presented in Figure 2(b).

For case $1\left(\Delta A_{i}=0\right)$, Figure 3 presents the time histories of the estimation errors, that is, $p=4$ components of the vector $e(t)=z(t)-\widehat{z}(t)$; from Figure 3 one remarks the canceling of the estimation errors, hence the effectiveness of the developed ROMO scheme. This is equivalent to the achievement of the state reconstruction (the convergences $\widehat{z}(t) \rightarrow z(t)$ and $\widehat{x}(t) \rightarrow x(t))$. The convergence speed of the ROMO (less than 2 seconds) represents an excellent convergence speed in the research area of the T-S multiple models.

For case $2\left(\Delta A_{i} \neq 0\right)$, Figure 4 presents again the time histories of the estimation errors' four components for different values of the scalars $\beta_{1}$ and $\beta_{2}$. One remarks the following: (1) the convergence time is approximately the same as in case of dynamics without uncertainties due to the robustness' improvement approach; (2) the increase of the scalars $\beta_{1}$ and $\beta_{2}$ does not modify the convergence time of ROMO; (3) the increase of the scalars $\beta_{1}$ and $\beta_{2}$ leads to the increase of the overshoots' absolute values for Figure 4(a) (from $7.1 \mathrm{~m} / \mathrm{s}$ for $\beta_{1}=\beta_{2}=0.8$ to $10.35 \mathrm{~m} / \mathrm{s}$ for $\beta_{1}=\beta_{2}=3.2$ ) and to the decrease of the overshoots' absolute values for Figure 4(b) (from $20.05 \mathrm{deg} / \mathrm{s}$ for $\beta_{1}=\beta_{2}=0.8$ to $18.35 \mathrm{deg} / \mathrm{s}$ for $\beta_{1}=\beta_{2}=3.2$ ).

Remark 6. One can make a brief comparison between the new designed reduced-order multiple observer and the only existing ROMO in the literature-the one designed in [19]. The first drawback of the observer from [19] is related to its existence conditions' number; thus, the observer from [19] has 4 existence conditions, while the one designing this paper has only one existence condition. The decrease of the existence conditions' number is due to the "while" loop which improves the generality character of the ROMO. The second main disadvantage of the reduced-order multiple observer from [19] is related to the usage of pole placement (eigenstructure assignment) method, the disadvantages of this technique being presented in the third section of the paper; it is possible that the choice of the observer's poles leads to the unwanted situation when $\lim _{t \rightarrow \infty} e(t) \neq 0$, this phenomena being called the eigenvalues' sensitivity; no robustness' improvement method is included in [19] for this possible problem. The third disadvantage of the observer from [19] with respect to the new ROMO from this paper is related to the lack of an analysis regarding the influence of uncertainties on the observer's performances. From the dynamic characteristics' point of view, the comparison between the 2 convergent reduced-order observers for T$S$ multiple models affected by unknown inputs cannot be done because different T-S multiple models have been used in the two papers; this is because two of the present ROMO's assumptions ((A3) and (A4)) do not allow the usage of the 


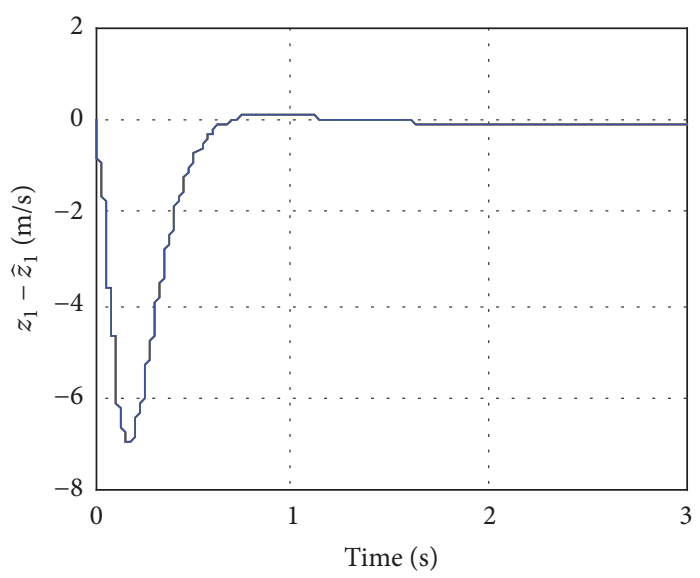

(a)

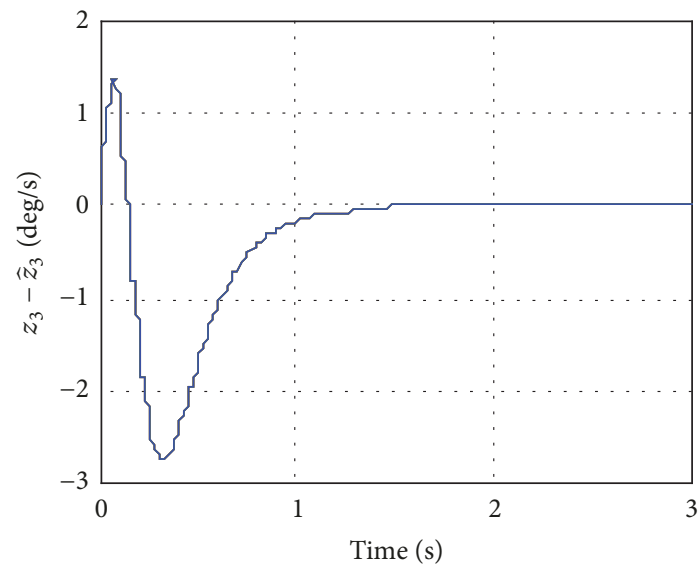

(c)

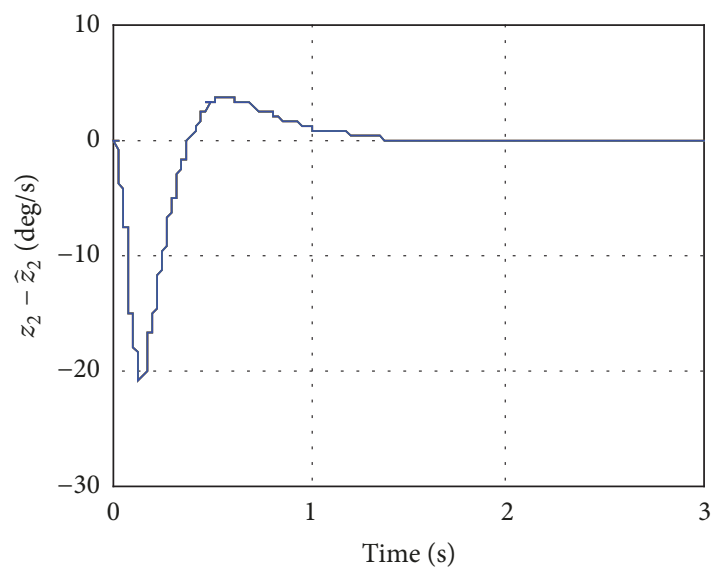

(b)

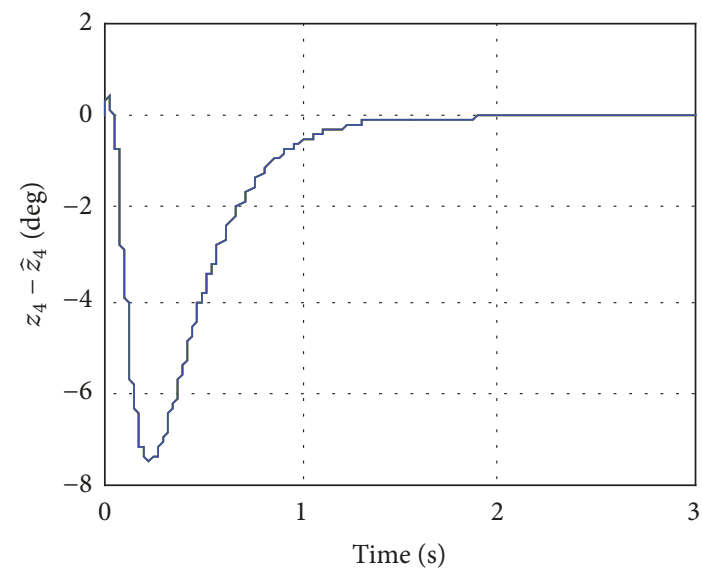

(d)

FIGURE 3: The new observer's estimation errors; case 1: dynamics without uncertainties.

Takagi-Sugeno multiple model from [19]. However, it has been shown that the new observer's convergence speed is an excellent one in the research area of the T-S multiple models. Taking into account all the above conclusions, one can remark the superiority of the new designed reduced-order multiple observer from existence conditions and robustness improvement point of view.

Remark 7. One can also make a brief comparison between the new designed ROMO and other existing FOMOs from the literature. Comparing to the state estimation error obtained by a method without taking into account the uncertainties (method proposed in [27]), one can conclude that the new proposed method is more robust against modeling uncertainties and unknown inputs. Another strong point of the new designed ROMO is the class of systems for which the state estimation is possible; the advantages of the used multiple models are related to the ability to extend the tools of the linear theory to nonlinear systems, to the possibility of reducing the complexity of nonlinear systems by constructing linear models that will be aggregated by using weighting functions, and to the fact that these aggregating functions possess the convexity property. Another advantage of the new ROMO with respect to other papers $([1,2])$ dealing with the design of FOMOs for T-S multiple models is related to the existence conditions which are less restrictive. Also, the new design procedure is effective because (1) it addresses the unknown inputs and the uncertainties decoupling problems in the same framework (and not as two independent problems); (2) it can be carried out using commercially available software, such as Matlab; (3) it provides all the degrees of design freedom which can be further utilized to achieve some additional system specifications; (4) the simulation results demonstrate superior convergence of the proposed ROMO compared to those in $[1,2,19]$.

\section{Conclusions}

The purpose of this study was to design a new reducedorder multiple observer, combining a typical reduced-order observer for LTI systems with unknown inputs and a fullorder multiple observer, to achieve the finite-time reconstruction of the system's state associated with T-S multiple models. The main advantage of the new designed ROMO is the decrease of the necessary sensors' number. To cancel the possible system uncertainties and to improve the observer 


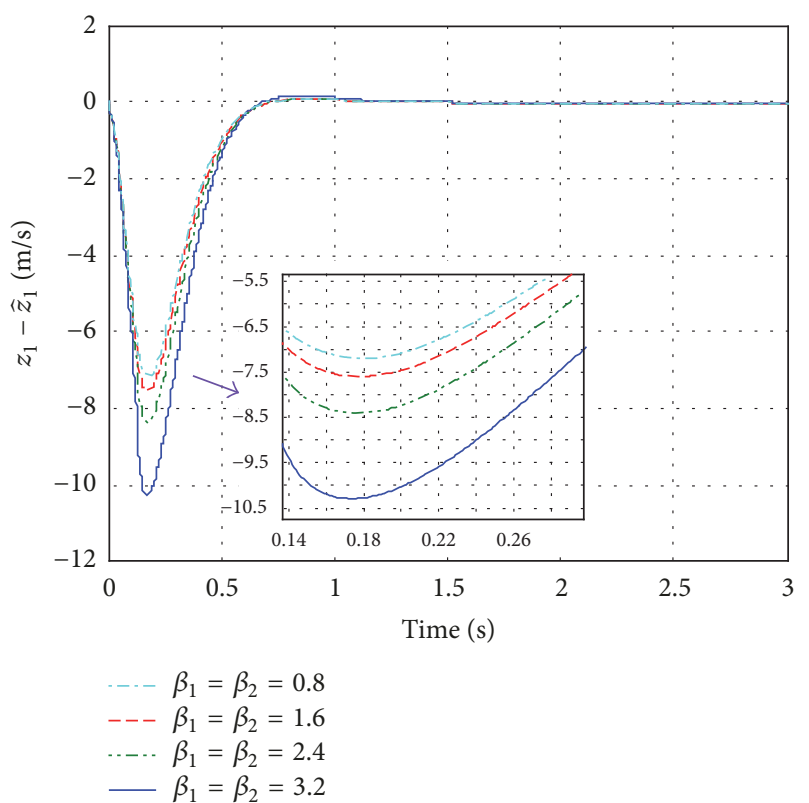

(a)

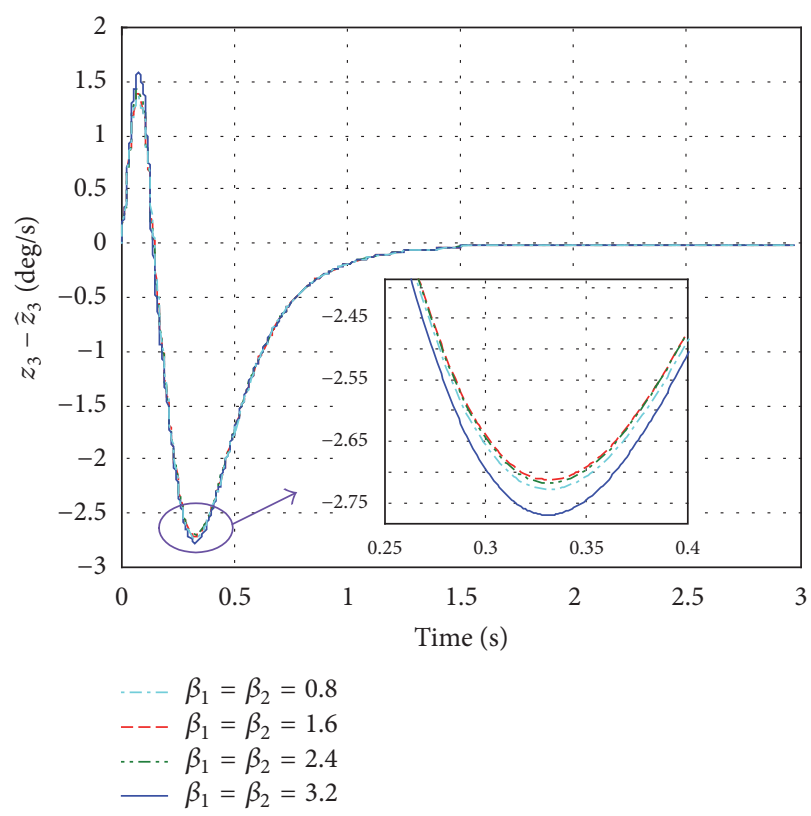

(c)

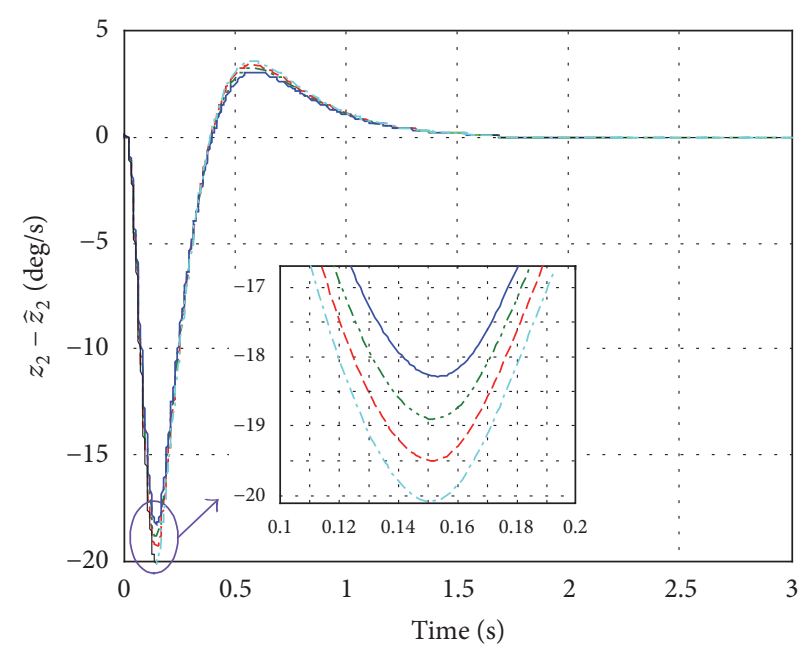

$\begin{aligned}-\beta_{1} & =\beta_{2}=0.8 \\ --\beta_{1} & =\beta_{2}=1.6 \\ \cdots-\cdots & \beta_{1}=\beta_{2}=2.4 \\ -\beta_{1} & =\beta_{2}=3.2\end{aligned}$

(b)

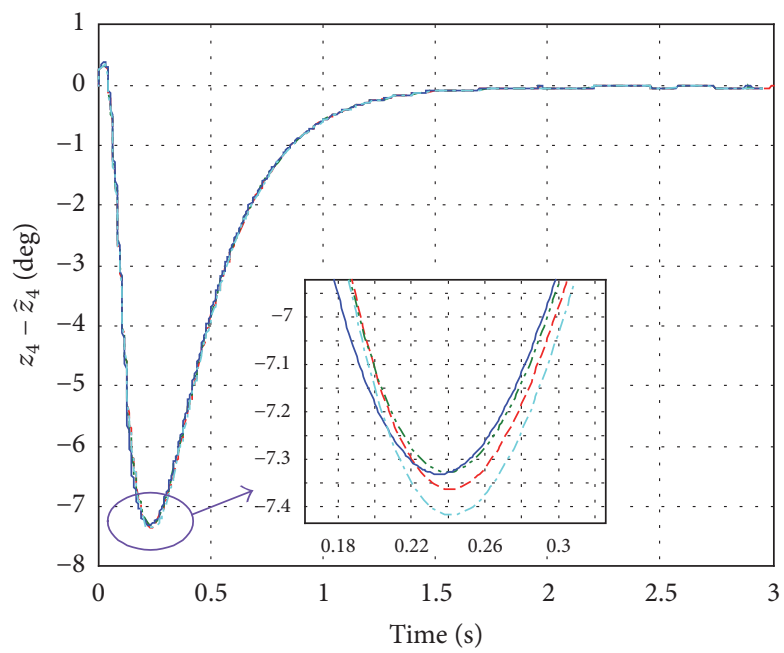

$\begin{aligned} \cdots & \beta_{1}=\beta_{2}=0.8 \\ \cdots-\beta_{1} & =\beta_{2}=1.6 \\ \cdots-\beta_{1} & =\beta_{2}=2.4 \\ -\beta_{1} & =\beta_{2}=3.2\end{aligned}$

(d)

FIGURE 4: The new observer's estimation errors; case 2: dynamics with uncertainties.

robustness, some compensation terms have been added in the observer design approach. To ensure the canceling of the state estimation errors, one has considered quadratic Lyapunov functions; one has been shown that the problem of reducedorder multiple observers for Takagi-Sugeno multiple models with unknown inputs can be reduced to the standard case when the unknown input vector does not interfere in the observer equations. The steps of the design procedure have been software implemented to validate the new ROMO; as an example, one has considered the lateral-directional motion of a light aircraft; the errors of the observer have been canceled even in the presence of unknown inputs and uncertainties. Future works will concern the application of these methods to the construct of robust ROMOs for T-S fuzzy systems with time delays.

\section{Conflicts of Interest}

The author declares that there are no conflicts of interest regarding the publication of this paper. 


\section{Acknowledgments}

This work was supported by a grant of the Ministry of National Education and Scientific Research, RDI Programme for Space Technology and Advanced Research (STAR), Project no. 181/20.07.2017.

\section{References}

[1] K. Bouassem, J. Soulami, A. El Assoudi, and E. H. El Yaagoubi, "Unknown input observer design for a class of Takagi-Sugeno descriptor systems," Nonlinear Analysis and Differential Equations, vol. 4, pp. 477-492, 2016.

[2] M. Chadli and H. R. Karimi, "Robust observer design for unknown inputs takagi-sugeno models," IEEE Transactions on Fuzzy Systems, vol. 21, no. 1, pp. 158-164, 2013.

[3] B. Aguiar, R. Márquez, and M. Bernal, "An LMI-based global non-quadratic observer design via Takagi-Sugeno models and Levant's robust differentiators," in Proceedings of the IEEE American Control Conference (ACC), pp. 119-124, Boston, MA, USA, 2016.

[4] M. Lungu and R. Lungu, "Design of full-order observers for systems with unknown inputs by using the eigenstructure assignment," Asian Journal of Control, vol. 16, no. 5, pp. 1470-1481, 2014.

[5] A. Buscarino, C. F. L. Fortuna, and M. Frasca, "Passive and active vibrations allow self-organization in large-scale electromechanical systems," International Journal of Bifurcation and Chaos, vol. 26, no. 7, Article ID 1650123, 2016.

[6] A. Buscarino, L. Fortuna, M. Frasca, and M. G. Xibilia, "Continuous time LTI systems under lossless positive real transformations: open-loop balanced representation and truncated reduced-order models," International Journal of Control, vol. 90, no. 7, pp. 1437-1445, 2017.

[7] A. Buscarino, L. Fortuna, M. Frasca, and M. G. Xibilia, "Positive-real systems under lossless transformations: Invariants and reduced order models," Journal of the Franklin Institute, vol. 354, no. 11, pp. 4273-4288, 2017.

[8] A. Akhenak, M. Chadli, D. Maquin, and J. Ragot, "State estimation of uncertain multiple model with unknown inputs," in Proceedings of the 43rd IEEE Conference on Decision and Control (CDC), pp. 3563-3568, Nassau, Bahamas, 2004.

[9] W. Jamel, A. Khedher, N. Bouguila, and K. B. Othman, "State estimation via observers with unknown inputs: Application to a particular class of uncertain takagi-sugeno systems," Studies in Informatics and Control, vol. 19, no. 3, pp. 219-228, 2010.

[10] L. Xiaohang, F. Zhu, A. Chakrabarty, and S. H. Żak, "Nonfragile fault-tolerant fuzzy observer-based controller design for nonlinear systems," IEEE Transactions on Fuzzy Systems, vol. 24, no. 6, pp. 1679-1689, 2016.

[11] D. Ichalal, B. Marx, J. Ragot, S. Mammar, and D. Maquin, "Sensor fault tolerant control of nonlinear Takagi-Sugeno systems. Application to vehicle lateral dynamics," International Journal of Robust and Nonlinear Control, vol. 26, no. 7, pp. 1376-1394, 2016.

[12] H. Li, C. Wu, S. Yin, and H. K. Lam, "Observer-Based fuzzy control for nonlinear networked systems under unmeasurable premise variables," IEEE Transactions on Fuzzy Systems, vol. 24, no. 5, pp. 1233-1245, 2016.

[13] K. Hfaiedh, K. Dahech, Y. Massaoudi, and T. Damak, "Sliding mode multiple observer for perturbed output nonlinear systems," in Proceedings of the 16th International Conference on
Sciences and Techniques of Automatic Control and Computer Engineering, STA 2015, pp. 740-745, Monastir, Tunisia, 2015.

[14] N. Bouguila, W. Jamel, A. Khedher, and K. B. Othman, "Multiple observer design for a non-linear Takagi-Sugeno system submitted to unknown inputs and outputs," IET Signal Processing, vol. 7, no. 8, pp. 635-645, 2013.

[15] K. Srinivasarengan, J. Ragot, D. Maquin, and C. Aubrun, "Nonlinear joint state-parameter observer for VAV damper position estimation," in Proceedings of the 3rd Conference on Control and Fault-Tolerant Systems, SysTol, pp. 164-169, IEEE, Barcelona, Spain, 2016.

[16] T. M. Guerra, R. Márquez, A. Kruszewski, and M. Bernal, "Ho LMI-Based Observer Design for Nonlinear Systems via Takagi-Sugeno Models with Unmeasured Premise Variables," IEEE Transactions on Fuzzy Systems, vol. PP, no. 99, p. 1, 2017.

[17] X. Su, P. Shi, L. Wu, and Y. Song, "A novel control design on discrete-time takagi-sugeno fuzzy systems with time-varying delays," IEEE Transactions on Fuzzy Systems, vol. 21, no. 4, pp. 655-671, 2013.

[18] X. Su, P. Shi, L. Wu, and S. Nguang, "Induced $\mathrm{L}_{2}$ filtering of fuzzy stochastic systems with time-varying delays," IEEE Transactions on Cybernetics, vol. 43, no. 4, pp. 1251-1264, 2013.

[19] M. Lungu and R. Lungu, "Reduced-Order Multiple Observer for Aircraft State Estimation during Landing," Applied Mechanics and Materials, vol. 841, pp. 253-259, 2016.

[20] T. Fernando, S. MacDougall, V. Sreeram, and H. Trinh, "Existence conditions for unknown input functional observers," International Journal of Control, vol. 86, no. 1, pp. 22-28, 2013.

[21] M. Lungu and R. Lungu, "Full-order observer design for linear systems with unknown inputs," International Journal of Control, vol. 85, no. 10, pp. 1602-1615, 2012.

[22] T. Takagi and M. Sugeno, "Fuzzy identification of systems and its applications to modeling and control," IEEE Transactions on Systems, Man, and Cybernetics, vol. 15, no. 1, pp. 116-132, 1985.

[23] T. Johansen and A. Foss, "Nonlinear local model representation for adaptive systems," in Proceedings of the International Conference on Intelligent Control and Instrumentation, IEEE, Singapore, 1992.

[24] M. Lungu and R. Lungu, Estimarea Stării Aparatelor De Zbor (State Estimation of the Flying Objects), Sitech Publisher, Craiova, Romania, 2014.

[25] M. Darouach, M. Zasadzinski, and S. J. Xu, "Full-order observers for linear systems with unknown inputs," IEEE Transactions on Automatic Control, vol. 39, no. 3, pp. 606-609, 1994.

[26] D. H. Lee and D. W. Kim, "Relaxed LMI conditions for local stability and local stabilization of continuous-time takagisugeno fuzzy systems," IEEE Transactions on Cybernetics, vol. 44, no. 3, pp. 394-405, 2014.

[27] V. Estrada-Manzo, Z. Lendek, and T. M. Guerra, "Unknown input estimation for nonlinear descriptor systems via LMIs and Takagi-Sugeno models," in Proceedings of the 54th IEEE Annual Conference on Decision and Control (CDC), pp. 6349-6354, IEEE, Osaka, Japan, 2015.

[28] S. Ahmadizadeh, J. Zarei, and H. R. Karimi, "A robust fault detection design for uncertain Takagi-Sugeno models with unknown inputs and time-varying delays," Nonlinear Analysis: Hybrid Systems, vol. 11, pp. 98-117, 2014.

[29] T. González and M. Bernal, "Progressively better estimates of the domain of attraction for nonlinear systems via piecewise Takagi-Sugeno models: Stability and stabilization issues," Fuzzy Sets and Systems, vol. 297, pp. 73-95, 2016. 


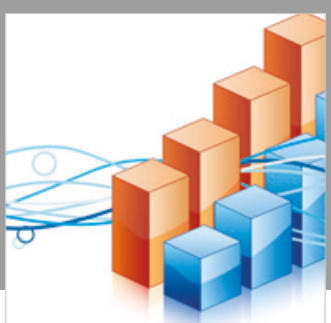

Advances in

Operations Research

\section{-n-m}
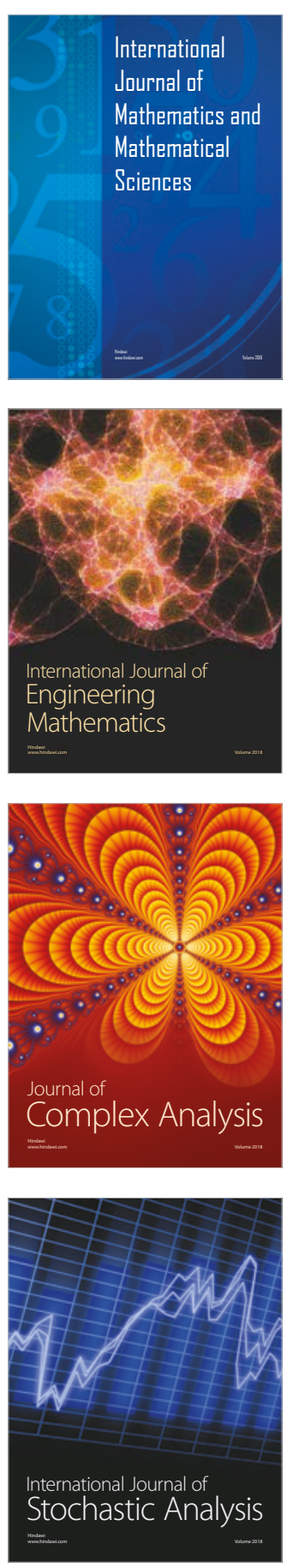
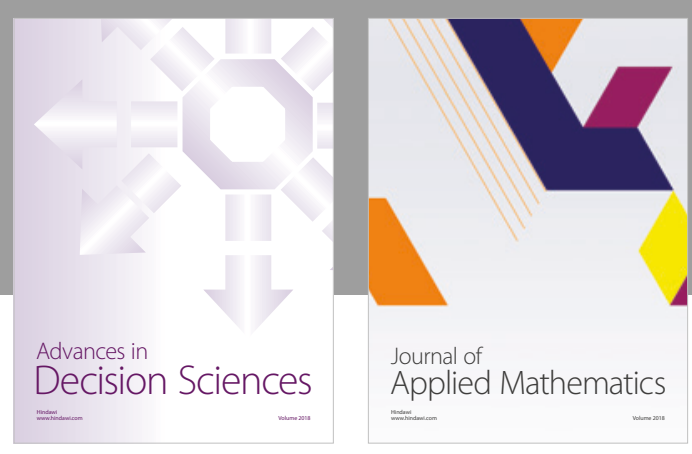

Journal of

Applied Mathematics
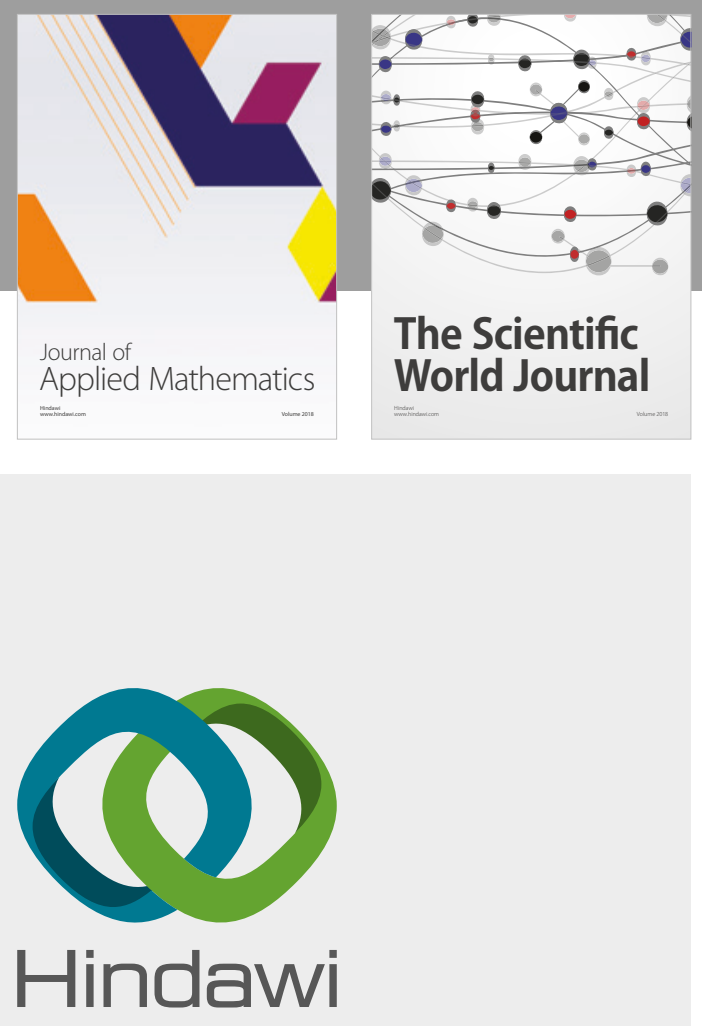

Submit your manuscripts at

www.hindawi.com

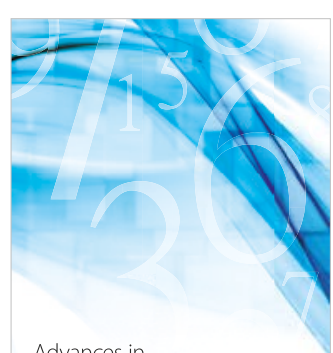

Advances in
Numerical Analysis
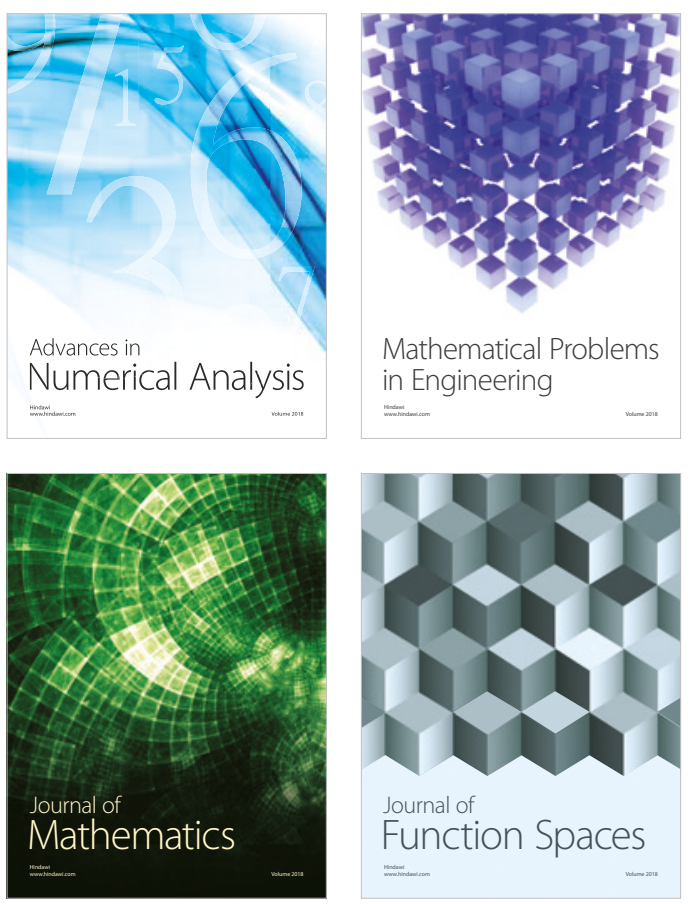

Mathematical Problems in Engineering

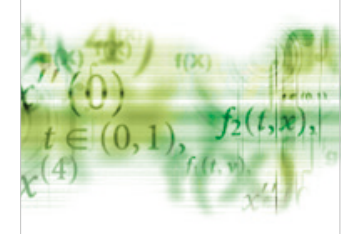

International Journal of

Differential Equations

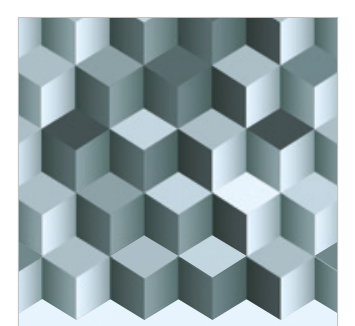

Journal of

Function Spaces
The Scientific

World Journal

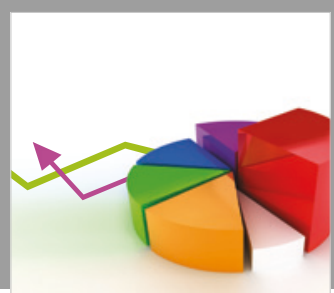

Journal of

Probability and Statistics
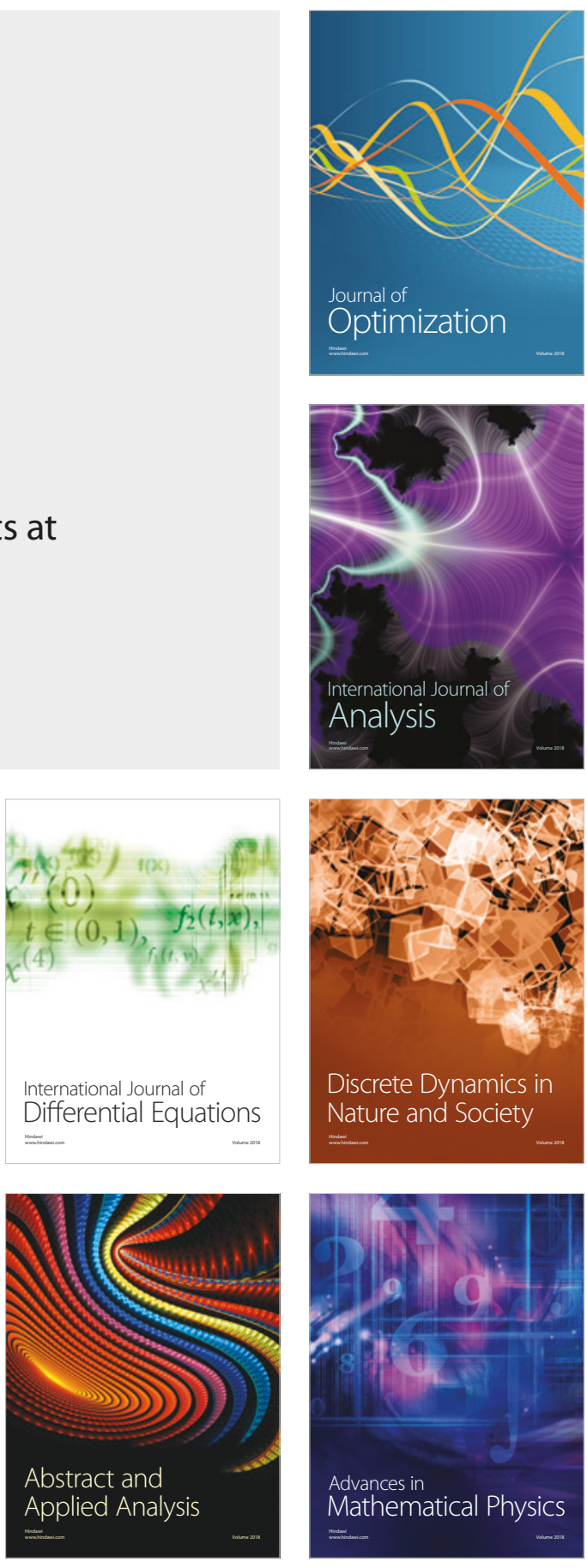